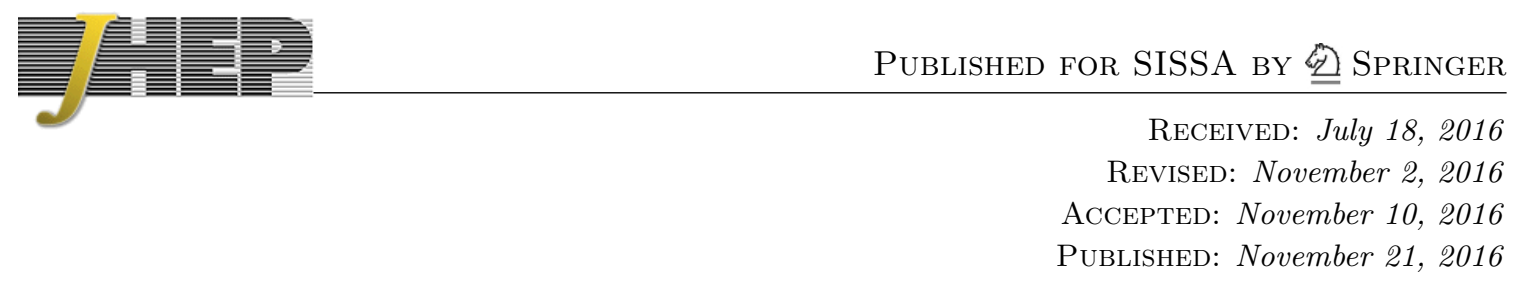

\title{
Capabilities of long-baseline experiments in the presence of a sterile neutrino
}

\author{
Debajyoti Dutta, ${ }^{a}$ Raj Gandhi, ${ }^{a}$ Boris Kayser, ${ }^{b}$ Mehedi Masud ${ }^{a}$ and \\ Suprabh Prakash ${ }^{a, c}$ \\ ${ }^{a}$ Harish-Chandra Research Institute, \\ Chhatnag Road, Jhunsi, Allahabad, 211019 India \\ ${ }^{b}$ Theoretical Physics Department, Fermilab, \\ P.O. Box 500, Batavia, IL, 60510 U.S.A. \\ ${ }^{c}$ School of Physics, Sun Yat-Sen (Zhongshan) University, \\ 135 Xingang Xi Road, Guangzhou, 510275 P.R. China \\ E-mail: debajyotidutta@hri.res.in, raj@hri.res.in, boris@fnal.gov, \\ masud@hri.res.in, prakash3@mail.sysu.edu.cn
}

ABStract: Assuming that there is a sterile neutrino, we ask what then is the ability of long-baseline experiments to i) establish that neutrino oscillation violates $\mathrm{CP}$, ii) determine the three-neutrino mass ordering, and iii) determine which $\mathrm{CP}$-violating phase or phases are the cause of any $\mathrm{CP}$ violation that may be observed. We find that the ability to establish $\mathrm{CP}$ violation and to determine the mass ordering could be very substantial. However, the effects of the sterile neutrino could be quite large, and it might prove very difficult to determine which phase is responsible for an observed $\mathrm{CP}$ violation. We explain why a sterile neutrino changes the long-baseline sensitivities to $\mathrm{CP}$ violation and to the mass ordering in the ways that it does. We note that long-baseline experiments can probe the presence of sterile neutrinos in a way that is different from, and complementary to, the probes of short-baseline experiments. We explore the question of how large sterile-active mixing angles need to be before long-baseline experiments can detect their effects, or how small they need to be before the interpretation of these experiments can safely disregard the possible existence of sterile neutrinos.

Keywords: Beyond Standard Model, CP violation, Neutrino Physics

ArXiv EPRINT: 1607.02152 


\section{Contents}

1 Introduction 1

2 Inputs and calculational procedures for the $3+1$ scenario 4

2.1 The $3+1$ mixing matrix 4

2.2 Simulation procedure 4

$\begin{array}{ll}2.3 \text { Details of experiments } & 6\end{array}$

3 Results and discussion $\quad 7$

3.1 Sensitivity to CP violation in the presence of a single sterile neutrino $\quad 7$

$\begin{array}{lll}3.2 & \text { Mass hierarchy } & 10\end{array}$

3.3 Using total event rates to understand the DUNE sensitivity to Hierarchy and CPV

3.4 To what extent can DUNE ignore the presence of the $3+1$ sector if it is present?

3.5 Determining the $\delta$ phase responsible for $\mathrm{CP}$ violation in $3+1$

3.6 How large do active-sterile mixings need to be before DUNE becomes sensitive to their presence?

\section{Introduction}

Much of the activity in neutrino oscillation experiments over the past two decades has focused on the increasingly precise determination of a) the neutrino mass-squared differences, $\delta m_{i j}^{2}=m_{i}^{2}-m_{j}^{2}$, and b) the mixing angles $\theta_{i j}$ along with the attendant CP phase $\delta_{\mathrm{CP}}$, which constitute the PMNS [1-3] mixing matrix $U_{\alpha i}$, with $i, j=1,2,3 \& i \neq j$ and $\alpha=e, \mu, \tau$. The complex matrix $U$ parameterizes the overlap between the neutrino mass eigenstates $\nu_{i}$ and the flavour eigenstates $\nu_{\alpha}$. While considerable progress has been achieved towards determining the mixing angles and mass differences to appreciable accuracy, the CP phase $\delta_{\mathrm{CP}}$ remains almost completely unknown.

The sources and qualitative nature of the experiments that have helped in this determination have been diverse, with neutrinos from the atmosphere, from reactors, from the sun and from particle accelerators being observed at detectors placed at baseline lengths spanning a wide range and employing a variety of detection techniques [4-16]. Global analyses [17-19] of the data collected have aided the consolidation of these efforts. This, in turn, has led to the gradual build-up of a consistent three family neutrino paradigm in impressive conformity with what is known, both theoretically and experimentally, about the lepton and quark sectors of the highly successful Standard Model (SM) of particle physics (when 
extended, nominally, to include massive neutrinos). Additionally, these experimental and theoretical efforts have helped formulate immediate questions that need to be answered by ongoing and planned experiments, which include the determination of a) the presence or absence of $\mathrm{CP}$ violation (CPV) in the lepton sector, and b) the mass hierarchy $(\mathrm{MH})$, or ordering, of the neutrino mass eigenstates.

Nonetheless, there are several ripples in this seemingly smooth fabric, at least some of which could be indicative of underlying new physics (for a discussion see [20]). Such physics, if real, could affect the interpretation and sensitivities of present and planned experiments. One such issue relates to signals from a variety of short-baseline experiments [21-25], which hint at the possible existence of short-wavelength oscillations, driven by one or more largely sterile states (i.e. states that do not have standard weak interactions, but couple indirectly via mixing) with $O\left(1 \mathrm{eV}^{2}\right)$ mass-squared splittings that are significantly larger than the two splittings that characterize the standard 3 family paradigm (referred to in what follows as the $3+0$ scenario). These short-wavelength oscillations can have significant effects when the (Baseline $L) /($ Energy $E$ ) of neutrinos in a beam is $\sim 1 \mathrm{~km} / \mathrm{GeV}$.

It is generally true, of course, that for significantly larger values of $L / E$, the shortwavelength oscillations driven by the large splittings involving an extra neutrino $\nu_{4}$ will be averaged to an L/E-independent value by the finite energy resolution of a typical detector. However, even the presence of a single additional sterile neutrino mass eigenstate (referred to as the $3+1$ scenario in what follows) introduces three additional mixing angles and two additional phases capable of significantly affecting oscillations at large baselines [26, 27]. It has been shown in [27], using the $1300 \mathrm{~km}$ Deep Underground Neutrino Experiment (DUNE) as an example, that if such a neutrino exists in nature, long-baseline results, interpreted without taking the short-wavelength oscillations into account, could erroneously imply that $\mathrm{CP}$ violation is very small or totally absent, when in reality it could be very large. In addition, measurements interpreted as determining the $\mathrm{CP}$-violating phase in the standard $3+0$ paradigm could in fact be measuring a linear combination of one or more phases belonging to the $3+1$ sector. These effects, which arise from large interference terms (between the $3+0$ and $3+1$ sectors) in the appearance probability, are accentuated by the presence of matter, which brings in contributions from sterile-sector mixings and phases that are quiescent at short baselines. Thus, the presence of the $3+1$ (or, more generally, 3 $+n$ ) sector can significantly blur any conclusions regarding CPV that may be drawn by long-baseline experiments.

Suppose that a $\sim 1 \mathrm{eV}$ mass largely-sterile neutrino does exist. What then is the ability of long-baseline experiments to establish that the leptonic weak interactions violate CP, to determine the mass-ordering of the three established neutrinos, and to determine which $\mathrm{CP}$-violating phase or phases is responsible for any $\mathrm{CP}$ violation that may be observed? In this paper, we address these questions. We find that the capacity to establish $\mathrm{CP}$ violation and to determine the mass ordering could be very substantial. However, the effects of the extra neutrino could be quite large, and could lead to erroneous or ambiguous conclusions, as already found in the earlier work [27]. We display examples that demonstrate the difficulty involved in determining which phase is causing an observed CP violation.

This work thus carries forward the approach adopted in [27]. Specifically, we study how expected sensitivities to the MH and CPV at NOvA [28], T2K [10], DUNE [29, 30] and 
HyperKamiokande (HK) [31] are altered in the $3+1$ scenario. Some of the other questions we attempt to answer are focused on DUNE as an example. For instance, suppose that the planned program of short-baseline experiments [32-35] that will probe the existence of $1 \mathrm{eV}$-mass sterile neutrinos does not see anything. We ask how tightly one must then bound the sterile-active mixing angles to ensure that DUNE data can be safely interpreted without taking the possible existence of sterile neutrinos into account.

Early work examining the effects of sterile neutrinos at long-baselines considered neutrino-factory experiments with baselines of $3000 \mathrm{~km}-7500 \mathrm{~km}$ and muon energies in the range of $20 \mathrm{GeV}-50 \mathrm{GeV}$, focusing on effects at both near and far detectors [36-40]. More recent work [26] includes a study of effects relevant to T2K [10] and a combined study [41] of T2K, MINOS [42] and reactor experiments which focuses on the effects of a $3+2$ scenario on $\theta_{13}$ measurements. Additionally, [43] has analysed the sensitivity of the experiment now known as DUNE to two sterile neutrinos whose physics is described by a minimal $3+2$ model. Recently, in [44] the long-baseline effects of one sterile neutrino have been studied, and sensitivity calculations in the presence of such a neutrino have been presented in $[45,46]$.

The analyses reported here differ from those referred to above in a number of ways, including physics content, approach, questions asked and underlying assumptions. While [43] considered a minimal $3+2$ model, the illustrative model we consider is $3+1$. [44] examines the large allowed range of $\Delta m_{41}^{2}$ and focuses on how well DUNE can constrain the $3+1$ and $3+0$ hypotheses assuming, in one case, that there is no sterile neutrino, and in a second case, if there is one sterile neutrino. In the $3+1$ scenario, in vacuum, only two effective CP phases out of three contribute (see, e.g. [27]). [44] estimates the sensitivity of the DUNE experiment to the new angles and the two effective phases in the $3+1$ scenario. Adding to the analyses in [44], we show how the sensitivity of long-baseline experiments to $\mathrm{CP}$ violation and the mass hierarchy depends on the new mixing angles and all three CP phases. Our work in this paper incorporates the fact that the presence of matter redefines the eigenstates, and quantitatively demonstrates that even those parameters which do not affect the $\nu_{\mu} \rightarrow \nu_{e}$ oscillations in vacuum can have significant effects in the presence of matter. In other words, it translates and extends, to the level of sensitivities, this important physics point regarding the effect of matter made earlier in [27], which demonstrated it at the level of probabilities and event rates. In [45, 46], the authors try to determine the potential of $\mathrm{T} 2 \mathrm{~K}, \mathrm{NO} \nu \mathrm{A}$ and $\mathrm{DUNE}$ to distinguish a $\mathrm{CP}$-violating value of a given $\mathrm{CP}$ phase $\delta_{x}$ from a situation where the same phase $\delta_{x}$ has been assumed to be CP conserving while leaving the other two phases free. In contrast to this, we determine the potential of the long-baseline experiments to distinguish between the situation where any one or more of the $\mathrm{CP}$ phases has a $\mathrm{CP}$-violating value, and the situation where all the $\mathrm{CP}$ phases have $\mathrm{CP}$-conserving values. In other words, to distinguish between the situation where $\mathrm{CP}$ is violated and the situation where it is not. We also try to provide an understanding of why the sensitivities to $\mathrm{CP}$ violation and the mass hierarchy behave as they do.

In section 2 below we define our mixing matrix parametrisation, describe our numerical simulation procedure and specify the constraints that motivate the parameter ranges we use in the simulation. We also provide brief descriptions and salient specifications for 
the long-baseline experiments considered in our work. Section 3 gives the results on mass hierarchy and $\mathrm{CP}$ sensitivities in the presence of a sterile neutrino and discusses the reasons why they differ substantially from the $3+0$ case. This section also addresses the question of how large sterile-active mixing angles need to be before measurable effects show up at long-baselines, and how small they need to be such that their presence can be safely ignored when interpreting the results of experiments. Section 4 provides the conclusions and summarizes the results of the paper.

\section{Inputs and calculational procedures for the $3+1$ scenario}

\subsection{The $3+1$ mixing matrix}

We begin by specifying our parameterisation for the PMNS matrix in the presence of a sterile neutrino;

$$
U_{\mathrm{PMNS}}^{3+1}=O\left(\theta_{34}, \delta_{34}\right) O\left(\theta_{24}, \delta_{24}\right) O\left(\theta_{14}\right) O\left(\theta_{23}\right) O\left(\theta_{13}, \delta_{13}\right) O\left(\theta_{12}\right) .
$$

Here, in general, $O\left(\theta_{i j}, \delta_{i j}\right)$ is a rotation matrix in the $i j$ sector with associated phase $\delta_{i j}$. For example,

$$
O\left(\theta_{24}, \delta_{24}\right)=\left(\begin{array}{cccc}
1 & 0 & 0 & 0 \\
0 & \cos \theta_{24} & 0 & e^{-i \delta_{24}} \sin \theta_{24} \\
0 & 0 & 1 & 0 \\
0 & -e^{i \delta_{24} \sin \theta_{24}} & 0 & \cos \theta_{24}
\end{array}\right) ; \quad O\left(\theta_{14}\right)=\left(\begin{array}{cccc}
\cos \theta_{14} & 0 & 0 & \sin \theta_{14} \\
0 & 1 & 0 & 0 \\
0 & 0 & 1 & 0 \\
-\sin \theta_{14} & 0 & 0 & \cos \theta_{14}
\end{array}\right) \text { etc. }
$$

In this parametrization, although the the vacuum $\nu_{\mu} \rightarrow \nu_{e}$ oscillation probability is independent of the 3-4 mixing angle and the associated CP phase, the presence of matter brings about a dependence on all mixing angles and phases. Specifically, unlike the vacuum case, the 3-4 mixing angle and its associated phase are no longer dormant, and the $3+1$ electron neutrino appearance probability at long baselines exhibits a significant dependence on them. Additionally, there are interference terms that enter for non-zero values of the phases that are not necessarily small, especially in the presence of matter, e.g., the term proportional to the sine of the sum of the phases $\delta_{13}$ and $\delta_{24}[27,47]$.

\subsection{Simulation procedure}

In this section, we describe the details of the simulation technique adopted in estimating the sensitivities and other results obtained. We have used the GLoBES [48, 49] software package for performing all our analyses. For extending the simulation to the $3+1$ scenario, we used $[50,51]$; which is an add-on to the default GLoBES software. Our assumptions regarding the values and ranges of the oscillation parameters for the $3+0$ sector are as follows:

- $\theta_{12}$ and $\theta_{13}$ are taken to be $33.48^{\circ}$ and $8.5^{\circ}$ respectively [52].

- $\delta m_{21}^{2}$ is taken to be $7.5 \times 10^{-5} \mathrm{eV}^{2}$ while $\delta m_{31}^{2}$ is set to be $2.457 \times 10^{-3} \mathrm{eV}^{2}(-2.374 \times$ $10^{-3} \mathrm{eV}^{2}$ ) for $\mathrm{NH}(\mathrm{IH})[52]$. 
- The currently-allowed $3 \sigma$ range on $\theta_{23}$ is $\left[38.3^{\circ}, 53.3^{\circ}\right]$ with the best fit at $42.3^{\circ}\left(49.5^{\circ}\right)$ for $\mathrm{NH}(\mathrm{IH})$ [52]. The $\theta_{23}$ best fit values from the global analyses $[19,53]$ are somewhat different from [52]. In this work, we make the simplifying assumption that 2-3 mixing is maximal; i.e. $\theta_{23}=45^{\circ}$. However, the conclusions we draw also apply to nonmaximal $2-3$ mixing.

It is anticipated that even if the $3+0$ scenario is not realised in nature, the above values and ranges will still hold to a very good approximation. ${ }^{1}$

We draw information regarding the value of $\delta m_{41}^{2}$ from [55], which does a combined analysis of the global data. The best fit of $\delta m_{41}^{2}$ in the $3+1$ scheme is found to be $0.93 \mathrm{eV}^{2}$ (table 8 of [55]). In our work, we assume $\delta m_{41}^{2}$ to be $+1 \mathrm{eV}^{2},{ }^{2}$ along with

$$
\delta m_{41}^{2} \sim \delta m_{42}^{2} \sim \delta m_{43}^{2} \gg\left|\delta m_{31}^{2}\right| \sim\left|\delta m_{32}^{2}\right| \gg \delta m_{21}^{2} .
$$

Our assumed ranges for the sterile sector mixing angles corresponding to the $3+1$ scenario draw upon current constraints and are as follows. Note that we derive the constraints correlated with the new mass-squared difference; i.e., the values of mixing angles that are compatible with $\delta m_{41}^{2} \sim 1 \mathrm{eV}^{2}$ are chosen.

- Measurements at the Daya Bay experiments put constraints on the effective mixing angle in the electron anti-neutrino disappearance channel. This effective mixing angle is the same as $\theta_{14}$ under the choice of PMNS parameterisation in this work. Based on [56], we assume $\theta_{14} \leq 13^{\circ}$ at $95 \%$ C.L. Slightly tighter constraints are available from the BUGEY experiment; but at $90 \%$ C.L. [57].

- The strongest constraints on the 2-4 mixing angle can be derived from the IceCube data [58]. With their current data, only $\theta_{24} \leq 7^{\circ}$ can be allowed at $99 \%$ C.L.

- The MINOS experiment with its observed charged-current and neutral-current events spectra can constrain the 3-4 mixing angle. From [59], we have $\theta_{34} \leq 26^{\circ}$ at $90 \%$ C.L.

We also vary $\delta_{13}, \delta_{24}$ and $\delta_{34}$ for $3+1$ and $\delta_{\mathrm{CP}}$ for $3+0$ over the full possible range of $\left[-180^{\circ}, 180^{\circ}\right.$. Finally, the fluxes we use are identical to those used in $[60] .{ }^{3}$ Details regarding the calculation of Poissonian $\chi^{2}$, treatment of systematic uncertainties etc. can be found in $[48,49]$.

\footnotetext{
${ }^{1}$ Some of our early calculations showed that the disappearance data at the far detector are less affected by the active-sterile mixing angles compared to the appearance data. Thus, the measurements that depend on $P_{\mu \mu}$, like $\sin ^{2} 2 \theta_{23}$ or $\left|\delta m_{31}^{2}\right|$, are expected to change less with the change of theoretical framework from $3+0$ to $3+1$. Likewise, it was shown in [54] that $\theta_{13}$ measurements at the reactor neutrino experiments will be robust even if there are sterile neutrinos. Also see [44] regarding this.

${ }^{2}$ The choice $\delta m_{41}^{2} \sim 1 \mathrm{eV}^{2}$ is made because it is a convenient benchmark. We have checked that our results and conclusions remain qualitatively valid for the mass splitting range $0.1-10 \mathrm{eV}^{2}$. Numerical differences in an important calculated quantity like the event rate, for instance, between our benchmark choice and the upper and lower bounds of this range are of the order of $10 \%$, and will bring about corresponding changes in calculations of sensitivities and other quantities calculated in our paper.

${ }^{3}$ These differ slightly in intensity and peak value from the present version used by the DUNE collaboration, but these differences do not affect our conclusions in any substantive manner.
} 


\subsection{Details of experiments}

T2K and T2HK. The Tokai to Kamioka (T2K) experiment is an ongoing neutrino experiment in Japan whose main goals are to observe $\nu_{\mu} \rightarrow \nu_{e}$ oscillations and to measure $\theta_{13}$. It may, however, be in a position to provide hints on CPV and the hierarchy, especially when its data are used in conjunction with those of other experiments. Neutrino beams generated at the J-PARC accelerator facility in Tokai are directed towards a $22.5 \mathrm{kton}$ water Črenkov detector placed in Kamioka, $295 \mathrm{~km}$ away at a $2.5^{\circ}$ off-axis angle [61]. The $\nu_{\mu}$ beam peaks sharply at $0.6 \mathrm{GeV}$, which is very close to the first oscillation maximum of the $\nu_{\mu} \rightarrow \nu_{e}$ appearance probability, $P_{\mu e}$. The flux falls off quite rapidly, such that it is negligible at energies greater than $1 \mathrm{GeV}$. The beam power is $750 \mathrm{~kW}$, with a proton energy of $30 \mathrm{GeV}$, for runs in both the $\nu$ and $\bar{\nu}$ modes. Combining both runs, the experiment will gather a total exposure of $\sim 8 \times 10^{21}$ protons on target (POT). The neutrino flux is monitored by the near detectors, located $280 \mathrm{~m}$ away from the point of neutrino production. Details regarding the detector efficiencies and background events used in our work have been taken from [48].

The T2HK experiment [31], in essence, is a scaled-up version of the T2K experiment. It will accumulate $1.56 \times 10^{22}$ protons on target with a $30 \mathrm{GeV}$ proton beam. The detector size is expected to be 25 times the T2K detector. For both T2K and T2HK, we have assumed 2.5\% (5\%) signal and 20\% (5\%) background normalisation errors in $\nu_{\mu}\left(\nu_{e}\right)$ signal. All details regarding signal and background events and detector efficiencies for T2HK are taken from $[31,62]$.

NO $\boldsymbol{\nu}$ A. The $\mathrm{NuMI}^{4}$ Off-axis $\nu_{e}$ Appearance experiment (NO $\left.\nu \mathrm{A}\right)$ [28] is an ongoing longbaseline super-beam experiment in the US. It aims to determine the mass hierarchy, $\theta_{13}$, the octant of $\theta_{23}$, and perhaps leptonic CP-violation, by the measurement of $\nu_{\mu} \rightarrow \nu_{e}$ oscillations. The source of $\nu_{\mu}$ is the Fermilab's NuMI beamline. A 14 kton Totally Active Scintillator Detector (TASD) is placed in Ash River, Minnesota, which is $810 \mathrm{~km}$ away at an off-axis angle of $14 \mathrm{mrad}\left(0.8^{\circ}\right)$. This off-axis narrow-width beam peaks at $2 \mathrm{GeV}$. The experiment is scheduled to run for 3 years in $\nu$ mode and 3 years in $\bar{\nu}$ mode with a NuMI beam power of $0.7 \mathrm{MW}$ and $120 \mathrm{GeV}$ proton energy, corresponding to $6 \times 10^{20}$ POT per year. A 0.3 kton near detector is located at the Fermilab site. We assume 5\% (2.5\%) signal normalisation error for $\nu_{e}\left(\nu_{\mu}\right)$ signal. Assumed background error is $10 \%$. The details of signal and background events and the detector efficiencies have been taken from [63].

DUNE. DUNE, a future experiment scheduled to come online $\sim 2025$, (with specifications very similar to LBNE $[29,30]$ ), will be located in the United States. It is a super-beam experiment with the main aim of establishing or refuting the existence of CPV in the leptonic sector. In addition to this primary goal, it will also be able to resolve the mass hierarchy and shed light on the octant of $\theta_{23}$. The $\nu_{\mu}\left(\bar{\nu}_{\mu}\right)$ super-beam will originate at Fermilab. The primary beam simulation assumes a $1.2 \mathrm{MW}-120 \mathrm{GeV}$ proton beam that will deliver $10^{21}$ protons-on-target (POT) per year. A 35-40 kt Liquid Argon (LAr) far-detector

\footnotetext{
${ }^{4}$ Neutrinos at the Main Injector.
} 

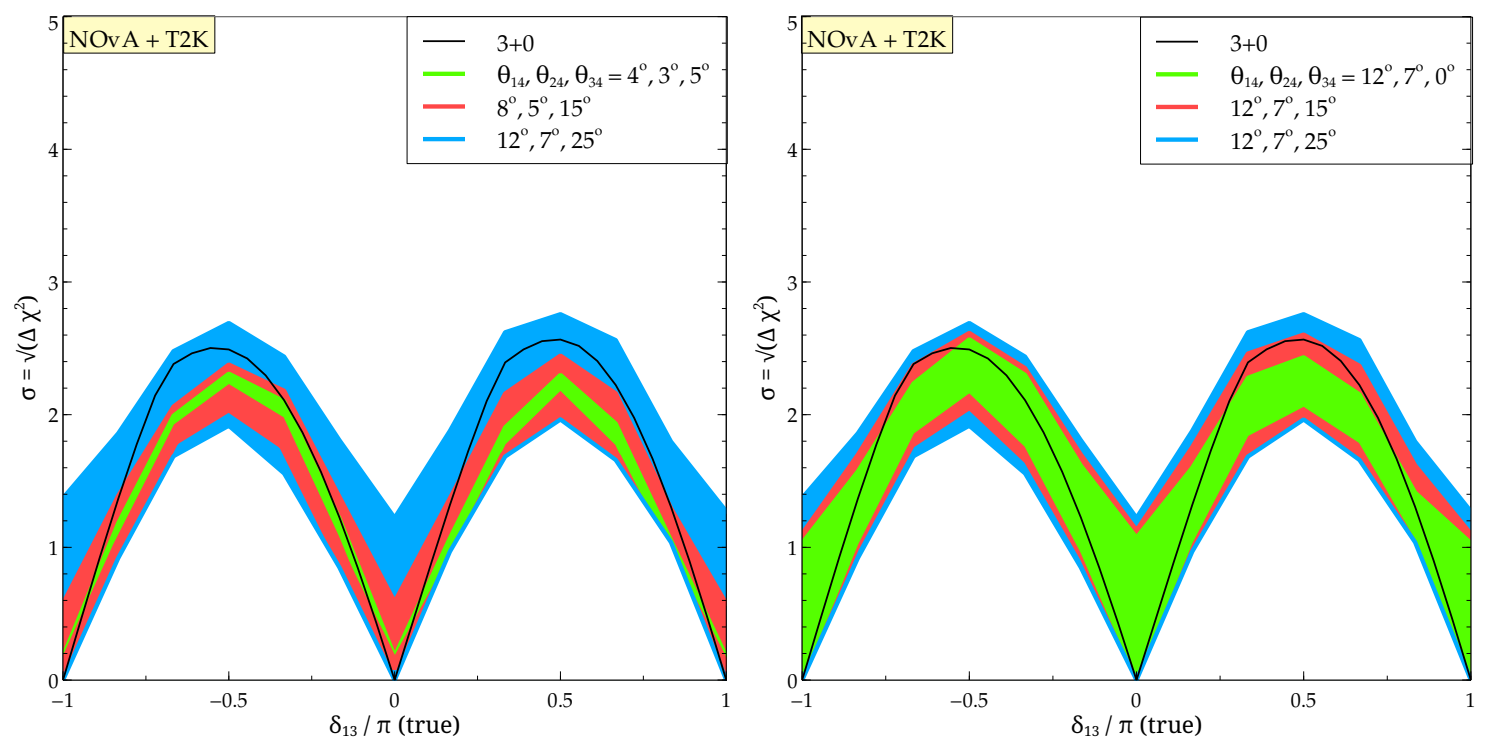

Figure 1. Sensitivity to $\mathrm{CP}$ violation as a function of the true $\mathrm{CP}$ violating phase $\delta_{13}$ for the combined data from $\mathrm{T} 2 \mathrm{~K}$ and $\mathrm{NO} \nu \mathrm{A}$. Different colors correspond to different choice of true $\theta_{14}, \theta_{24}, \theta_{34}$ as shown in the key. Variation of true $\delta_{24}$ and $\delta_{34}$ results in the colored bands which show the minimum and maximum sensitivity that can be obtained for a particular $\delta_{13}$. The black curve corresponds to sensitivity to $\mathrm{CP}$ violation in $3+0$. Left panel: shows the effect as all the three active-sterile mixings are increased. Right panel: shows the effect of the 3-4 mixing when the true $\theta_{14}$ and $\theta_{24}$ have been fixed at $12^{\circ}$ and $7^{\circ}$ respectively for all three bands.

will be housed in the Homestake mine in South Dakota, $1300 \mathrm{~km}$ away. ${ }^{5}$ The experiment plans to have a total of 10 years of running, divided equally between neutrinos and antineutrinos, corresponding to a total exposure of $35 \times 10^{22} \mathrm{kt}$-POT-yr. Other experimental details, such as signal and background definitions as well as the detector efficiencies taken in this work are the same as those in [60], except with the difference that we have not considered tau events in the backgrounds. We assume a $5 \%$ signal normalisation error and a $10 \%$ background normalisation error. The detector efficiencies for both $P_{\mu e}$ and $P_{\bar{\mu} \bar{e}}$ events are close to $80 \%$ with somewhat less efficiency for $P_{\bar{\mu} \bar{e}}$.

\section{Results and discussion}

\subsection{Sensitivity to CP violation in the presence of a single sterile neutrino}

In this section, we show the sensitivities of various long-baseline experiments to leptonic CP violation in the $3+1$ scenario. Results for the $3+0$ case are provided for comparison. Figures 1 to 4 show the sensitivity to excluding the $\mathrm{CP}$ conserving values as a function of the $3+1$ oscillation parameters.

We show results for three sets of the active-sterile mixing angles $\theta_{14}, \theta_{24}, \theta_{34}$. These are chosen to be $\left(4^{\circ}, 3^{\circ}, 5^{\circ}\right),\left(8^{\circ}, 5^{\circ}, 15^{\circ}\right)$ and $\left(12^{\circ}, 7^{\circ}, 25^{\circ}\right)$. The data are simulated assuming

\footnotetext{
${ }^{5}$ In addition to this, there is a proposal to install a near detector [64, 65], which among other physics goals can also constrain the parameter space for the $3+1$ scenario, as recently discussed in [66].
} 

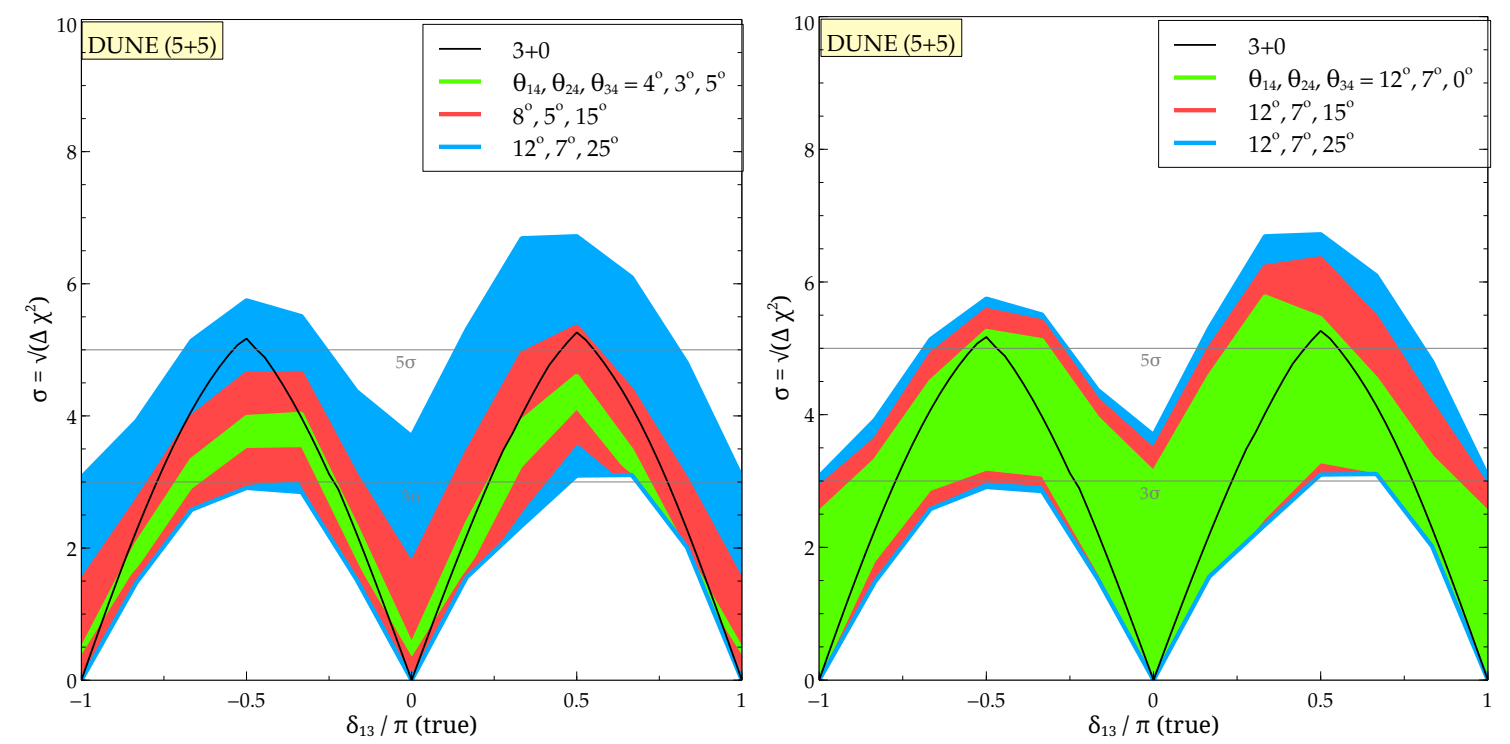

Figure 2. Similar to figure 1 but for DUNE. The hierarchy is assumed to be normal.

the above three sets of mixing angles and various choices of the three $\mathrm{CP}$ phases lying in $\left[-180^{\circ}, 180^{\circ}\right]$. In the fit, we consider the 8 possible $\mathrm{CP}$ conserving combinations of $\delta_{13}, \delta_{24}, \delta_{34}$ where each phase could either be 0 or $180^{\circ}$. We minimise the $\Delta \chi^{2}$ over these 8 test $\mathrm{CP}$ conserving cases; and over a relatively fine grid of test $\theta_{14}, \theta_{24}, \theta_{34}$ samples in the allowed range in the fit, so as to account for the lack of information regarding activesterile mixings. This gives us a $\Delta \chi_{\min }^{2}$ as a function of the true parameters. In creating figures 1 to 3 , both for simulating data and in the fit, we assume the hierarchy to be normal only (we will consider an inverted hierarchy shortly). We did not marginalise over the $3+0$ parameters except $\delta_{13} \cdot{ }^{6}$ For a particular true $\delta_{13}$, we show the maximum and the minimum $\Delta \chi_{\min }^{2}$ that can be obtained corresponding to a variation of the other two true CP phases $\delta_{24}$ and $\delta_{34}$. For $3+0$, the situation is simpler, where we contrast a true $\delta_{\mathrm{CP}}$ against $\delta_{\mathrm{CP}}=0$ and $\delta_{\mathrm{CP}}=\pi$ in the fit.

It can be seen from figures 1 to 3 that the existence of sterile neutrinos can significantly affect the CPV discovery potential of long-baseline experiments. This violation can originate in any of the three phases and not just $\delta_{13}$. When the active-sterile mixings are small, the general trend visible in the figures is that the sensitivity to $\mathrm{CP}$ violation of the experiment will be decreased compared to what we would expect in the $3+0$ scenario. However, for sufficiently large mixings, the sensitivity spans both sides of the $3+0$ curve; and hence, depending on the true value of the other phases $-\delta_{24}$ and $\delta_{34}$, the sensitivity to $\mathrm{CP}$ violation can be greatly amplified. We observe that the chosen value of true $\theta_{34}$ significantly affects the sensitivity to CP violation, especially for DUNE, where the matter

\footnotetext{
${ }^{6}$ Our results show that a close to $5 \sigma$ determination of hierarchy is very likely with the DUNE experiment, even in the $3+1$ paradigm. Among other $3+0$ oscillation parameters, marginalisation over $\theta_{23}$ may be important when the non-maximal true values like the ones in lower octant or higher octant are considered because of octant-related degeneracies. Since, disappearance $\left(P_{\mu \mu}\right)$ data fixes $\sin ^{2} 2 \theta_{23}$ very accurately, marginalisation over test $\theta_{23}$ is not necessary for true $\theta_{23}=45^{\circ}$.
} 

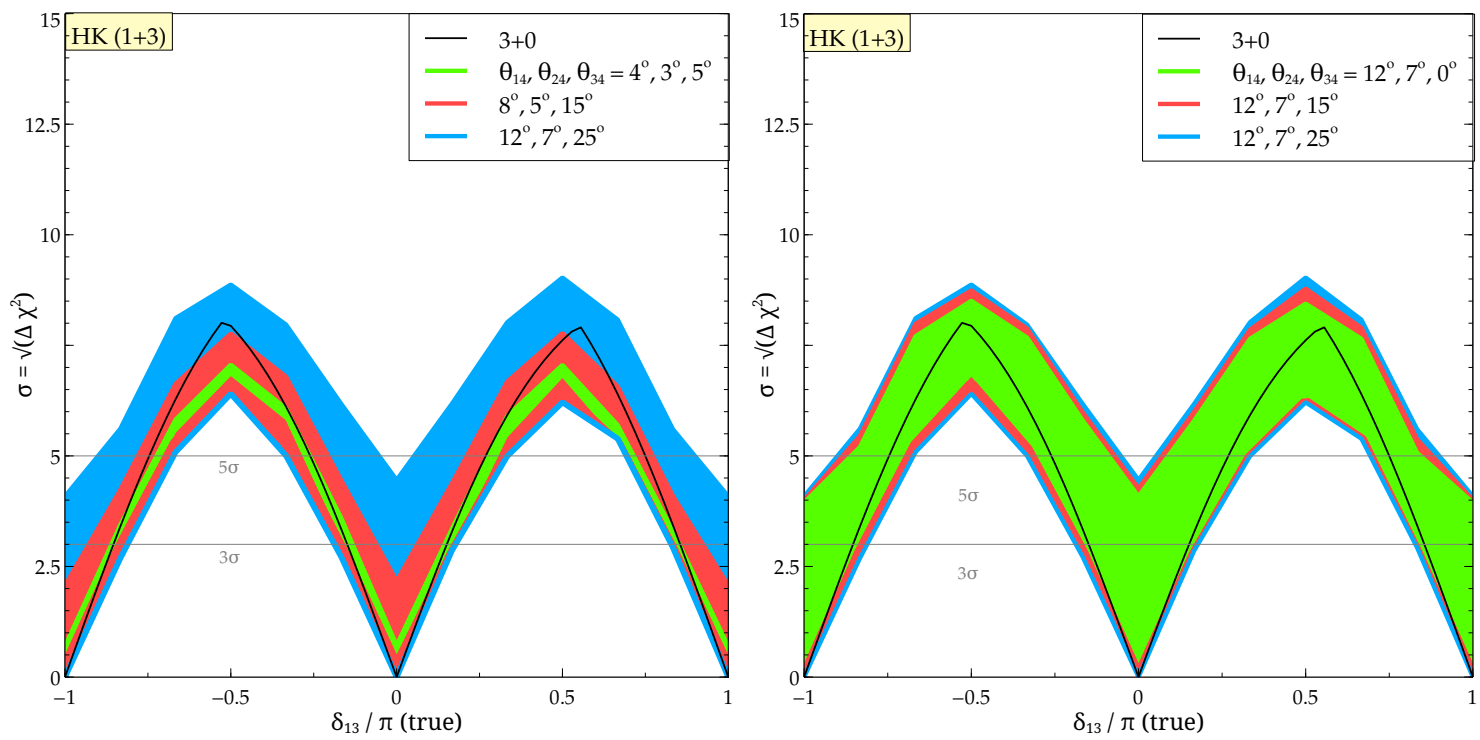

Figure 3. Similar to figure 1 but for T2HK.
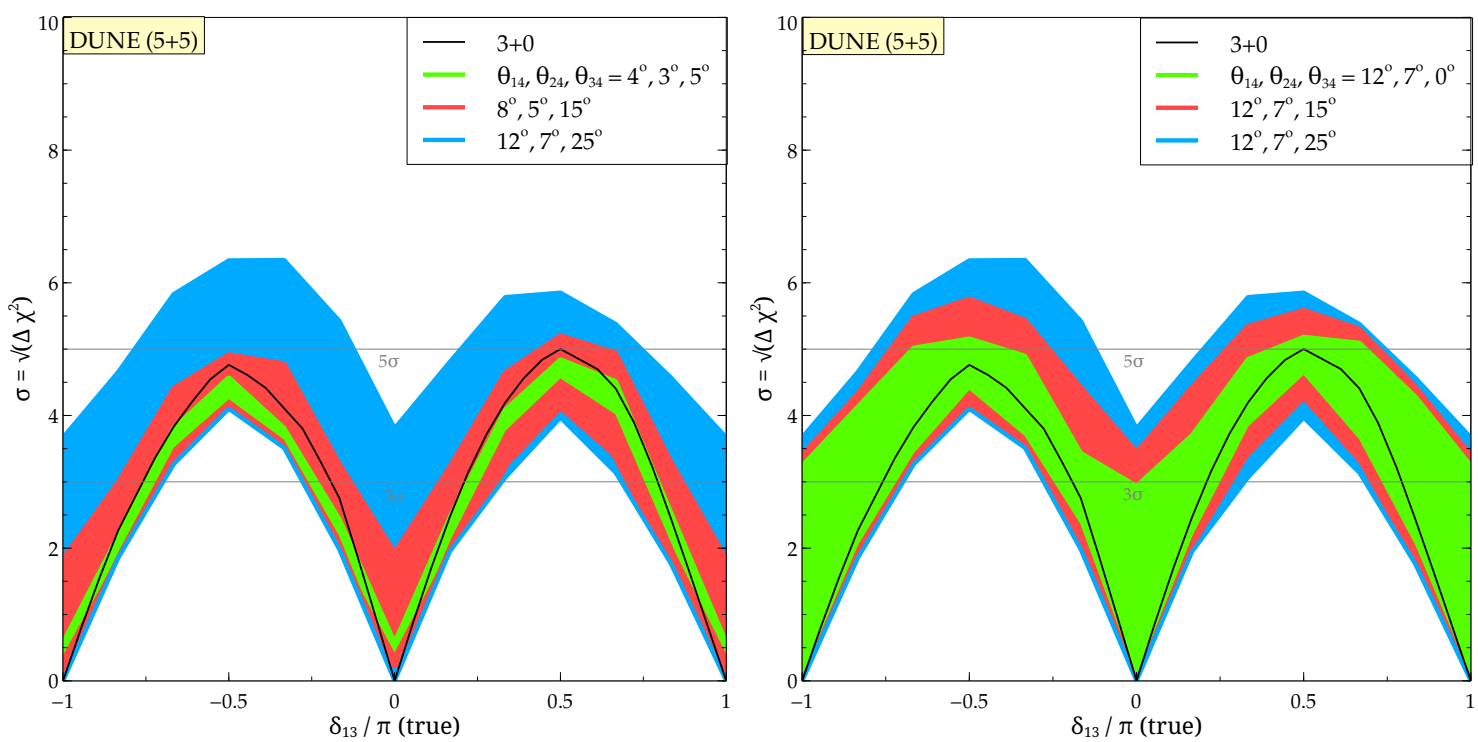

Figure 4. Similar to figure 2 but for inverted hierarchy (IH).

effects are large. We also note that there can be significant amplification of CPV sensitivity for regions of $\delta_{13}$ where one expects little or none in the $3+0$ scenario.

Similar observations can also be made when the true hierarchy is considered to be inverted. As an example, we have shown in figure 4 the CPV sensitivity results for DUNE (similar to figure 2) for inverted hierarchy and the features are qualitatively similar.

Overall, if sterile mixing angles are not tiny, the $3+1$ (or $3+n)$ scenario, if realized in nature, makes the observation of generic CPV per se significantly more likely than the $3+0$ case, although it makes the determination of the phase (or phases) in which such violation originates much harder (we address this point in greater detail in subsection 3.5). 
In order to understand the behavior of sensitivities to $\mathrm{CP}$ violation and later the mass hierarchy, we note the role of the following competing effects in the calculation of $\Delta \chi_{\min }^{2}:^{7}$

1. The parameter space for the $3+1$ scenario consists of $\theta_{14}, \theta_{24}, \theta_{34}, \delta_{24}, \delta_{34}$, and $\delta m_{41}^{2}$, in addition to the standard $3+0$ parameters. While generating the $\Delta \chi_{\min }^{2}$ curve for $3+0$ scenario (black), we marginalized only over the test parameter $\delta_{13}$ (i.e. $\delta_{\mathrm{CP}}$ ); however, for the $3+1$ curves, marginalization was carried over the five additional test parameters $\theta_{14}, \theta_{24}, \theta_{34}, \delta_{24}, \delta_{34}$ in addition to $\delta_{13}$. Hence, the parameter space for $3+1$ case is a substantially larger superset of the $3+0$ parameter space. In general, from a statistical point of view, marginalization over a larger space of test parameters tends to bring down the value of $\Delta \chi_{\min }^{2}$.

2. As the true values of the $3+1$ mixing angles $\theta_{14}, \theta_{24}, \theta_{34}$ increase, the impact due to the variation in the true values of the associated phases $\left(\delta_{24}, \delta_{34}\right)$ also increases. This results in substantially broadening the $\Delta \chi_{\min }^{2}$ bands (for $3+1$ ) in both directions as the true values of $\theta_{14}, \theta_{24}, \theta_{34}$ increase from $4^{\circ}, 3^{\circ}, 5^{\circ}$ to $12^{\circ}, 7^{\circ}, 25^{\circ}$, - thereby making the $\Delta \chi_{\min }^{2}$ (for $3+1$ scenario) sometimes even larger than the $3+0 \Delta \chi_{\min }^{2}$.

When the active-sterile mixing angles (true $\theta_{14}, \theta_{24}, \theta_{34}$ ) are small, effect (2) is small. However, the statistical effect (effect (1) above) stemming from marginalization over five added parameters is significant and reduces the sensitivity in general. Consequently, $\Delta \chi_{\min }^{2}$ tends to decrease for small values of the true sterile mixing angles. On the other hand, when the mixing angles (true $\theta_{14}, \theta_{24}, \theta_{34}$ ) increase, they concurrently amplify the effect of the $\mathrm{CP}$ violating phases. Consequently, effect (2) plays a correspondingly important role, and tends to increase $\Delta \chi_{\min }^{2}$ overall. These features have been explained in greater detail in subsection 3.3 using the DUNE total neutrino and anti-neutrino event rates as an example.

\subsection{Mass hierarchy}

In this section, we re-evaluate the sensitivities of the long-baseline experiments to the neutrino mass hierarchy, assuming the $3+1$ scenario. We first show results assuming the normal hierarchy. The simulation procedure followed here is the same as that described in section 3.1 except for the following important differences:

- In the fit, we assume the hierarchy to be inverted.

- For the CP violation sensitivities, in the fit, we had considered only those combinations of test values of $\delta_{13}, \delta_{24}, \delta_{34}$ which were CP conserving. For hierarchy determination, however, we have varied these test $\mathrm{CP}$ phases in their full allowed range $\left[-180^{\circ}, 180^{\circ}\right]$ and have marginalised over them.

Note that, as in the previous section, we did not vary the test $3+0$ parameters other than the $\mathrm{CP}$ phase in the fit. We show the results in figures 5 and 6 as a function of the true $3+1$ parameters. For the combined results from $\mathrm{T} 2 \mathrm{~K}$ and $\mathrm{NO} \nu \mathrm{A}$, it can be seen in figure 5

\footnotetext{
${ }^{7}$ Similar effects can also guide the value of $\Delta \chi_{\min }^{2}$ in the context of other new physics effects such as NSI and this has been illustrated in [67, 68].
} 

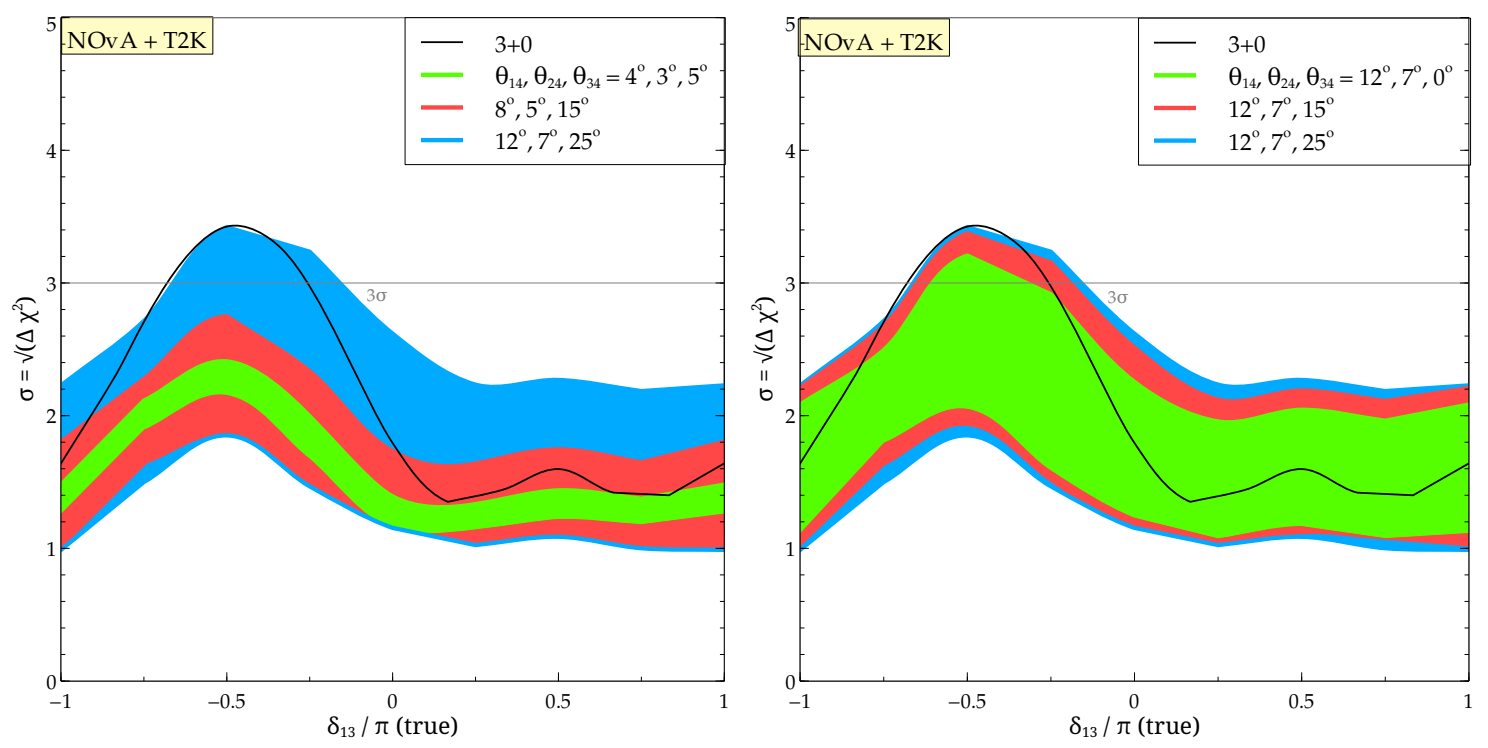

Figure 5. Sensitivity to Mass hierarchy as a function of the true $\mathrm{CP}$ violating phase $\delta_{13}$ for the combined data from $\mathrm{T} 2 \mathrm{~K}$ and $\mathrm{NO} \nu \mathrm{A}$. Different colors correspond to different choice of true $\theta_{14}, \theta_{24}, \theta_{34}$ as shown in the key. Variation of true $\delta_{24}$ and $\delta_{34}$ results in the colored bands which show the minimum and maximum sensitivity that can be obtained for a particular $\delta_{13}$. The black curve corresponds to sensitivity to the hierarchy in $3+0$. Left panel: shows the effect as all the three active-sterile mixings are increased. Right panel: shows the effect of the 3-4 mixing when the true $\theta_{14}$ and $\theta_{24}$ have been fixed at $12^{\circ}$ and $7^{\circ}$ respectively for all three bands.

(left panel) that there emerges the possibility of significant improvement in the hierarchy sensitivity compared to $3+0$ (shown by black line) in the unfavourable regions of true $\delta_{13}$. The extent of this enhancement is, of course, dependent on the true values of the activesterile oscillation parameters. Figure 5 (right panel) shows the dependence of sensitivity on the $\theta_{34}$ mixing angle - the effects of which are noticeable, although not large, even for baselines where matter effects are not very substantial.

In the case of DUNE, we find that the $3+1$ sensitivities are usually below $3+0$ sensitivities $^{8}$ except for a small region of parameter space around true $\delta_{13}=90^{\circ}$, as can be observed in both the panels of figure 6 . The $\theta_{34}$-dependence of sensitivities is somewhat more pronounced for DUNE, as is evident from the right panel of figure 6. It should be noted that except for a small fraction of parameter space around true $\delta_{13}=90^{\circ}$, the sensitivity stays above $5 \sigma$ C.L.

In figure 7 , we have shown the mass hierarchy sensitivity of DUNE when the true hierarchy is inverted. The positions of the peaks and troughs of the sensitivities are opposite to those in figure 6 . The general features of the sensitivities are qualitatively similar to those corresponding to a normal true hierarchy (as in figure 6), except for the following

\footnotetext{
${ }^{8}$ As mentioned in the beginning of the present subsection 3.2, the test CP phases have been marginalized over their full allowed range $\left[-180^{\circ}, 180^{\circ}\right]$ while calculating the $\Delta \chi_{\min }^{2}$ for mass hierarchy; whereas for CP violation sensitivity the marginalization was carried over the CP-conserving values $-0^{\circ}$ and $180^{\circ}$ only. This enhanced range of marginalization for mass hierarchy leads to a larger statistical effect (see effect (1) in the previous subsection), leading to a generally reduced $\Delta \chi_{\min }^{2}$ for $3+1$, compared to the $3+0$ case.
} 

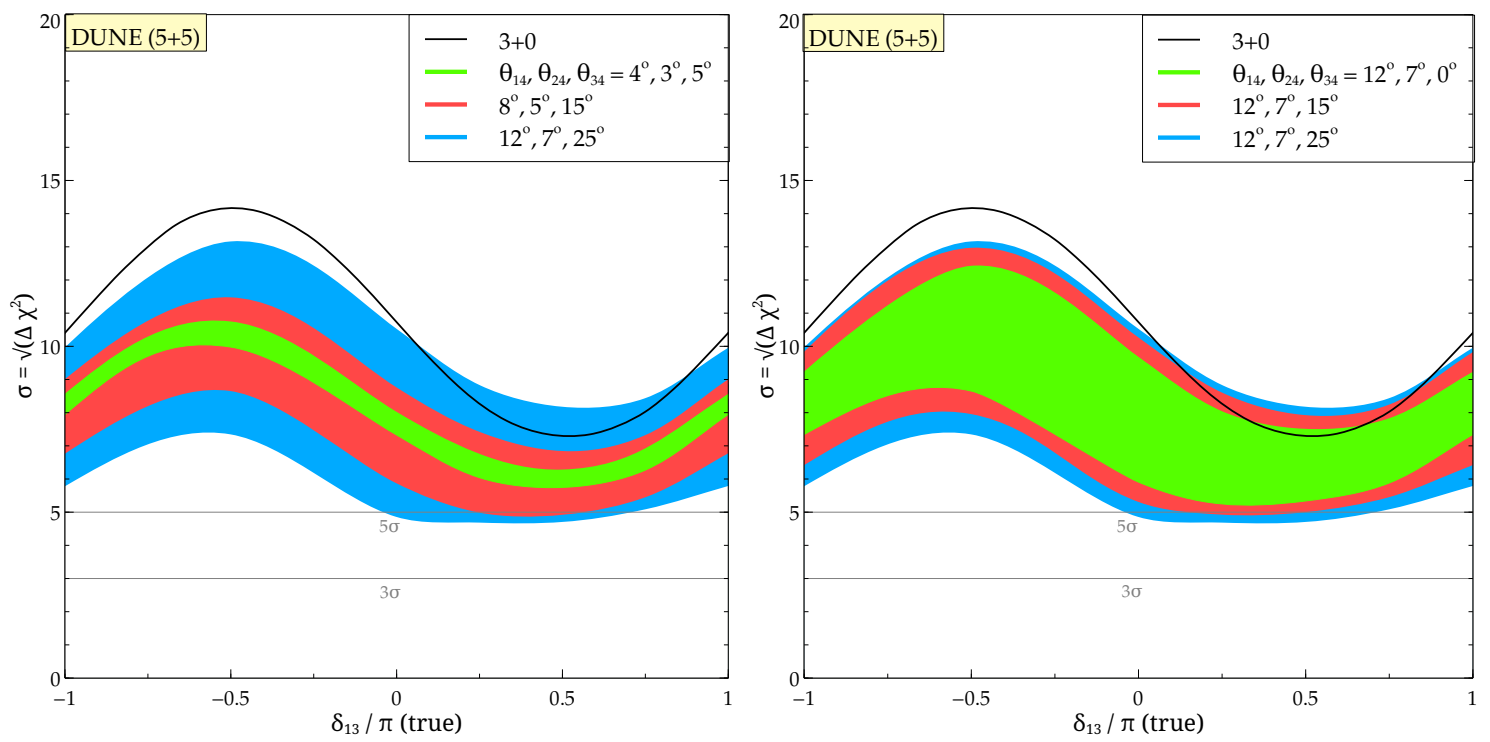

Figure 6. Similar to figure 5 but for DUNE. Normal hierarchy was considered to be true.
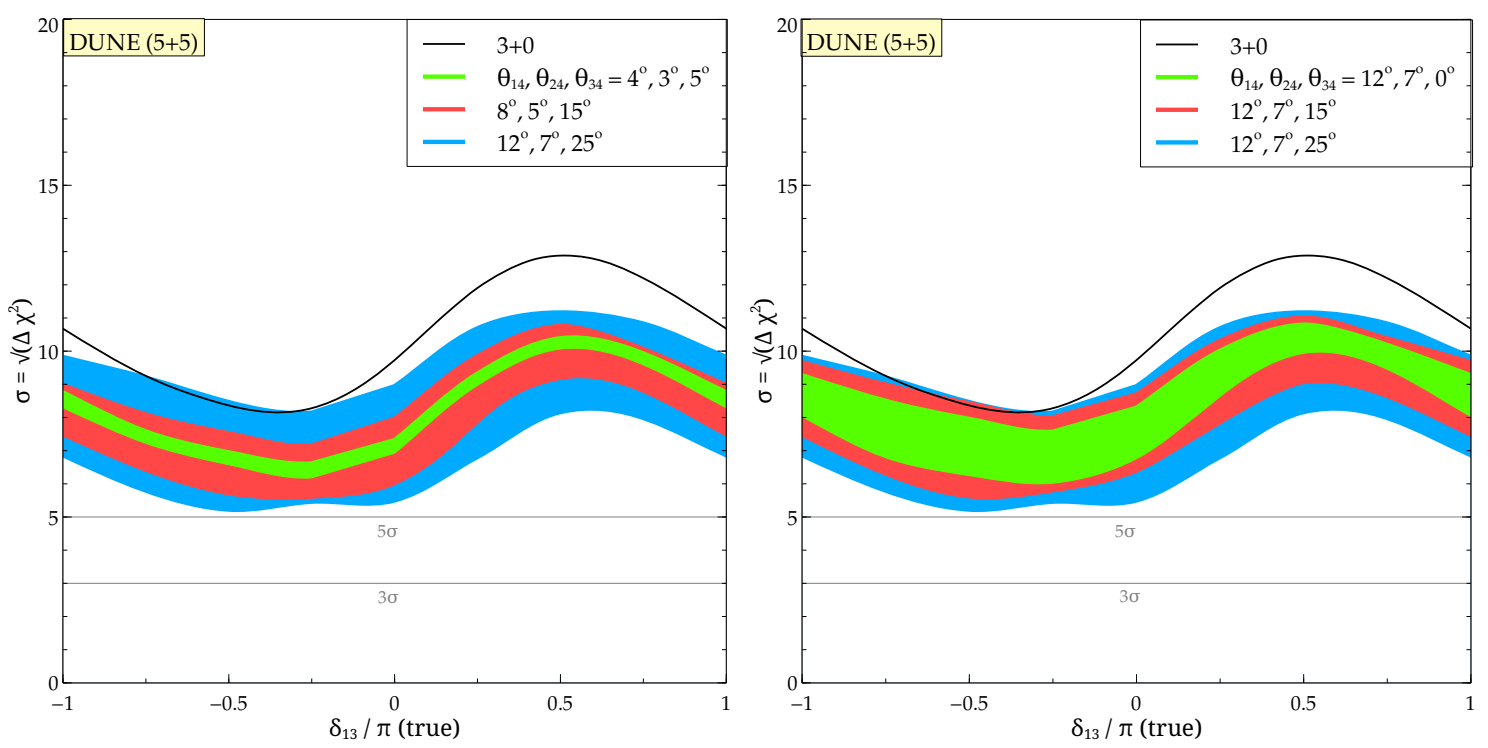

Figure 7. Similar to figure 6 but when the true hierarchy is considered to be inverted.

small difference: - the hierarchy sensitivity bands in figure 7 in the presence of a sterile neutrino are slightly narrower than those in figure 6 . The sensitivities in the presence of a sterile neutrino remain above the $5 \sigma$ C.L.

\subsection{Using total event rates to understand the DUNE sensitivity to Hierarchy and CPV}

This subsection attempts to obtain a better understanding of the changes that occur in $\mathrm{CP}$ and hierarchy sensitivities at long baselines (as seen in subsections 3.1 and 3.2) in the presence of a sterile neutrino. It provides, in effect, an alternative way of looking at the 

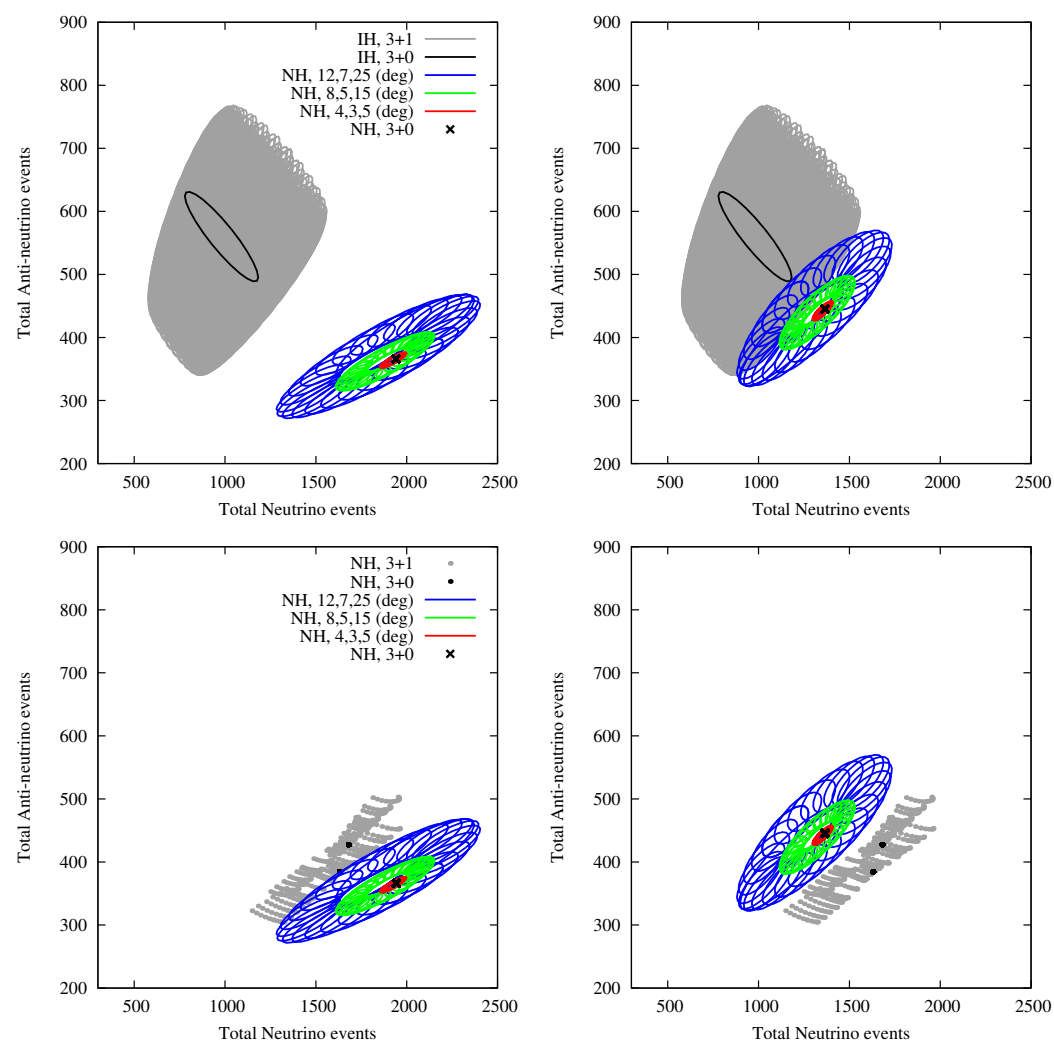

Figure 8. Neutrino and Anti-neutrino event rates for the DUNE experiment. The top panels are to explain the observed hierarchy sensitivity while the bottom panels are for CPV. The event rates in blue, green and red correspond to the simulated data with $\mathrm{NH}$ and the active-sterile mixings as depicted in the keys. The black crosses correspond to true event rates for $\mathrm{NH}$ and $\delta_{\mathrm{CP}}=-90^{\circ}\left(90^{\circ}\right)$ assuming $3+0$ for the left (right) panel. The event rates in grey in the top panel correspond to the fit events rates assuming $3+1$ where all the active-sterile oscillation parameters have been varied. The event rates in grey in the bottom panel correspond to fit event rates assuming $3+1$ where the active-sterile mixing angles have been varied and each of the three CP phases is either 0 or $\pi$. In the top (bottom) panels, the assumed hierarchy for fit events is $\mathrm{IH}(\mathrm{NH})$. In the top panels, the black ellipses correspond to fit events with only $\delta_{\mathrm{CP}}$ varied assuming $3+0$. In the bottom panels, the black dots correspond to fit events with $\delta_{\mathrm{CP}}=0, \pi$ assuming $3+0$.

qualitative behaviour exhibited by the sensitivities in figures 1 through 6 and complements the discussion in the subsections above. We use DUNE as our primary example, but the features discussed below are, in general, displayed in the figures corresponding to other experiments as well, e.g. those for NOvA + T2K or for HK.

In figure 8 , we try to explain the results that we obtained for the hierarchy and $\mathrm{CP}$ violation sensitivities of the DUNE experiment using total (energy-integrated) neutrino and anti-neutrino event rates. The top panels of figure 8 refer to the hierarchy sensitivity behavior while the bottom panels are for $\mathrm{CP}$ violation. The regions shown in red, green, blue and the black cross correspond to true oscillation parameters (or the data). The regions in grey, the black dots and the black ellipses correspond to the test oscillation parameters (or the fit). 
The data events on the left plots are for true $\mathrm{NH}$ and true $\delta_{13}=-90^{\circ}$ while the data events on the right plots are for true $\mathrm{NH}$ and true $\delta_{13}=90^{\circ}$. The grey fit events in the top panels correspond to test IH and all $3+1\left(\theta_{14}, \theta_{24}, \theta_{34}, \delta_{24}, \delta_{34}\right.$ in addition to $\left.\delta_{13}\right)$ parameters varied, while the black ellipse in the top panel corresponds to test IH and variation of $\delta_{\mathrm{CP}}$ only, assuming $3+0$. The grey fit events in the bottom panel correspond to test $\mathrm{NH}$ and test CP-conserving combinations of $\delta_{13}, \delta_{24}, \delta_{34}$, while the black dots in the bottom panel correspond to test $\mathrm{NH}$ and test $\delta_{\mathrm{CP}}=0, \pi$; assuming $3+0$. Broadly, it can be said that the sensitivities will be better if the data events and the fit events are well separated (ignoring sensitivity coming from spectral information). The sensitivity patterns can be understood if we focus on the following two features:

- The fit event rates region increases drastically as we go from $3+0$ to $3+1$ (black ellipses and dots compared to grey regions). Note that we marginalise over the entire allowed $3+1$ oscillation parameter space.

- For small mixing angles $\theta_{i 4}$, the event rate regions are small compared to their size for large mixing angles (red compared to green and blue).

Thus, we can make the following deductions which are reflected in the sensitivity plots.

- Compared to $3+0$, the 3+1-small mixing angles reduce the sensitivity of DUNE to hierarchy or $\mathrm{CP}$ violation because although the increase in the data event regions is small, the fit event regions cover significantly more area, decreasing the separation between the two types of regions, and consequently, the sensitivity. This is visible in the green regions in the left panels of figures 2 and 6.

- As we go from small mixing angles to large mixing angles, the area covered by the data event rates increases significantly. Thus, there are oscillation parameters for which the data events are either very far or very close to the fit event regions compared to $3+0$. Consequently, the $3+1$ sensitivities can be much better or much worse compared to the $3+0$. This is demonstrated, for instance, by the blue regions in the left panels of figure 2 (both better and worse; for $\mathrm{CP}$ ) and figure 6 (mostly worse; for hierarchy).

- Figure 8 also makes it easier to understand the behavior with respect to true $\delta_{13}$. As we go from $\delta_{13}=-90^{\circ}$ (left plots) to $\delta_{13}=90^{\circ}$ (right plots), the fit rates approach data rates in the case of hierarchy and hence the sensitivity decreases, as seen in the coloured regions of both the left and right panels of figure 6 . However, in the case of $\mathrm{CP}$ violation, the fit rates are symmetrically placed around the data region and therefore, the sensitivity remains roughly the same in both quadrants, as is manifested in the left and right panels of figure 2 .

\subsection{To what extent can DUNE ignore the presence of the $3+1$ sector if it is present?}

In this subsection we explore the question whether the CP-related measurements at DUNE can get affected by assuming the absence of sterile neutrinos when they exist in reality, 

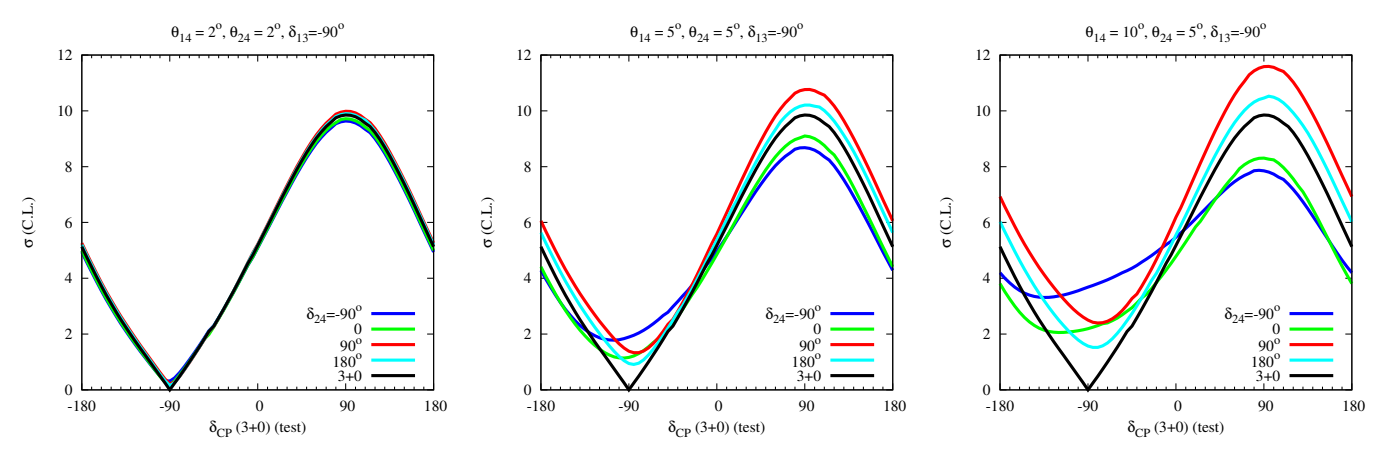

Figure 9. $\sigma=\sqrt{\Delta \chi^{2}}$ as a function of test $\delta_{\mathrm{CP}}$ for the DUNE experiment. We contrast $3+1$ as the true case with $3+0$ as the test case. Results are shown for different $3+1$ oscillation parameters. These plots are for true $\delta_{13}=-90^{\circ}$. True $\delta_{24}$ values have been varied as shown in the key. The left, middle and right panels correspond to true $\theta_{14}=\theta_{24}=2^{\circ}, \theta_{14}=\theta_{24}=5^{\circ}$ and $\theta_{14}=10^{\circ}, \theta_{24}=5^{\circ}$ (i.e. $\sin ^{2} 2 \theta_{\mu e}$ equal to $6 \times 10^{-6}, 2 \times 10^{-4}$ and $9 \times 10^{-4}$ ) respectively. We assume $\theta_{34}=\delta_{34}=0$. Both true as well as test hierarchy is assumed to be normal. The black curve shows the usual sensitivity when both true and test are $3+0$.

but are obscure because of very small mixings. To explore this point in greater detail, we carry out the following exercise. We assume that the true scenario is $3+1$ with sufficiently small active-sterile mixings such that $\sin ^{2} 2 \theta_{\mu e} \leq 10^{-3}$, i.e. below the currently stated sensitivity of short-baseline experiments planned or underway at Fermilab [32-35]. For such small values of $\theta_{14}$ and $\theta_{24}, \theta_{34}$ is rendered inconsequential in $P_{\mu e}$; therefore, we assume $\theta_{34}=\delta_{34}=0$ for simplicity. We choose several true values of $\delta_{13}$ and $\delta_{24} \in\left[-180^{\circ}, 180^{\circ}\right]$. In the fit, we assume $3+0$ and vary $\delta_{\mathrm{CP}} \in\left[-180^{\circ}, 180^{\circ}\right]$, calculating the sensitivity at each test $\delta_{\mathrm{CP}}$. We fix the remaining $3+0$ oscillation parameters in the fit at their true values. The results are shown in figure 9, and the details of our assumptions and chosen values are as described in the figure caption.

It is possible to obtain the following information from the curves in figure 9:

- They show the allowed test $\delta_{\mathrm{CP}}$ region. For example, in the $3+0$ scenario, DUNE should be able to measure $\delta_{\mathrm{CP}}$ with a precision of $\pm 60^{\circ}$ at $3 \sigma$ C.L. if true $\delta_{\mathrm{CP}}=-90^{\circ}$.

- They demonstrate the sensitivity at which the experiment can exclude $\mathrm{CP}$ conservation i.e. test $\delta_{\mathrm{CP}}=0,180^{\circ}$ if the true $\delta_{\mathrm{CP}}=-90^{\circ}$.

- Finally, they help answer the question: can $3+0$ be excluded if $3+1$ is true? If for all test $\delta_{\mathrm{CP}}$ values, $\Delta \chi^{2}>\mathrm{N}^{2}$, then we can say that $3+0$ can be excluded at $\mathrm{N} \sigma$.

We observe in figure 9 that if $\theta_{14}$ and $\theta_{24}$ are as small as $2^{\circ}$ each (left panel), then the results obtained are essentially the same even if the wrong theoretical framework of $3+0$ is considered while fitting the data. For angles as large as $5^{\circ}$ (middle panel), there are visible but not highly significant deviations from the $3+0$ results. Depending on the true value of $\delta_{24}$, the precision with which DUNE measures $\delta_{13}$ can improve or worsen by $\sim 10^{\circ}$. However, the predicted best fit is around the right test $\delta_{\mathrm{CP}}\left(=-90^{\circ}\right)$ in most cases. An offset, of $\sim 15^{\circ}$, is obtained depending on the true value of $\delta_{24}$. For the $3+0$ case, DUNE 

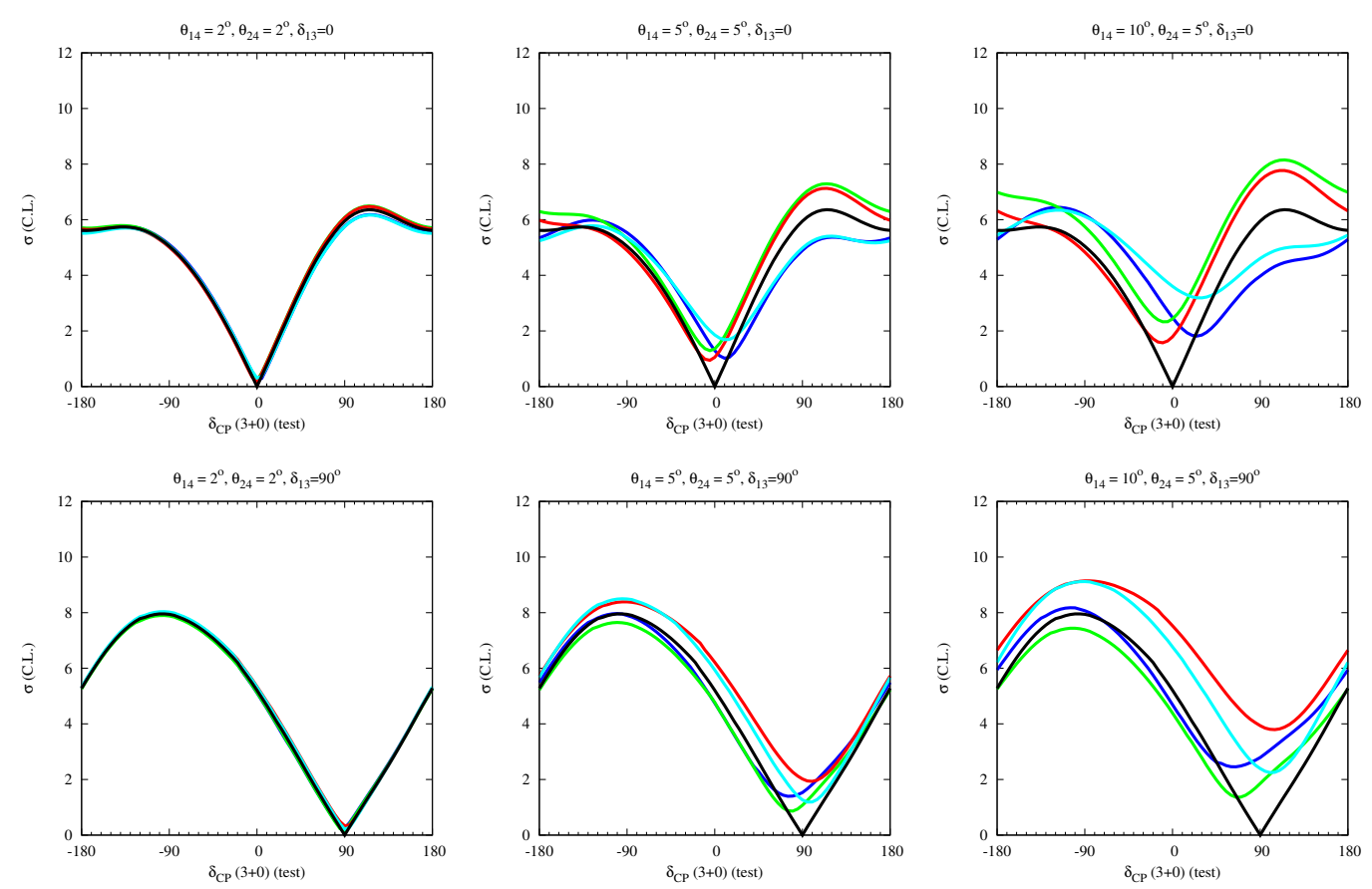

Figure 10. Same as figure 9 , but for $\delta_{13}=0$ (top panel) and $\delta_{13}=90^{\circ}$ (bottom panel).

can exclude $\mathrm{CP}$ conserving test $\delta_{\mathrm{CP}}=-180^{\circ}$ at $5 \sigma$. This sensitivity, in the $3+1$ case, can vary from $4-6 \sigma$. For $\theta_{14}=\theta_{24}=5^{\circ}$, DUNE can exclude $3+0$ at $1-2 \sigma$ depending on the true value of $\delta_{24}$. For still larger values of mixing angles like $\theta_{14}=10^{\circ}, \theta_{24}=5^{\circ}$, we observe similar features except that the $\Delta \chi^{2}$ are larger i.e. DUNE is more likely to exclude $3+0$.

This exercise has been repeated for true $\delta_{13}=0$ and $90^{\circ}$ in figure 10 , with similar results.

\subsection{Determining the $\delta$ phase responsible for CP violation in $3+1$}

Suppose that the results of short-baseline experiments indicate the presence of a $\sim 1 \mathrm{eV}$ neutrino, and, in addition, DUNE finds evidence of CP violation. Assuming this situation, we attempt to examine the extent and accuracy with which DUNE can identify the source of this violation, i.e. the particular $3+1$ phase (i.e. $\delta_{13}, \delta_{24}$ or $\delta_{34}$ ) associated with it. To simplify matters, we assume near-maximal-allowed values of the sterile mixing angles; i.e. $\left(\theta_{14}, \theta_{24}, \theta_{34}\right):\left(12^{\circ}, 7^{\circ}, 25^{\circ}\right)$ and also fix the true value of $\delta_{34}$ to 0 , in order to better bring out the effect of the other two CP phases.

Results are shown for the following four combinations of true $\left(\delta_{13}, \delta_{24}\right):\left(0, \pm 90^{\circ}\right)$ and $\left( \pm 90^{\circ}, 0\right)$. These correspond to the situation where one of the phases is maximally $\mathrm{CP}$ violating, while the other is $\mathrm{CP}$ conserving. In the fit, we marginalised over the test activesterile mixing angles and the test $\mathrm{CP}$ phase $\delta_{34}$. The $2 \sigma, 3 \sigma$ and $4 \sigma$ allowed regions in the test $\delta_{13}$ - test $\delta_{24}$ plane are shown in figure 11 for the above-mentioned four true combinations (these plots can also be used to find out the precision with which DUNE can measure the CP phases; see $[44,46]$ for such results). 

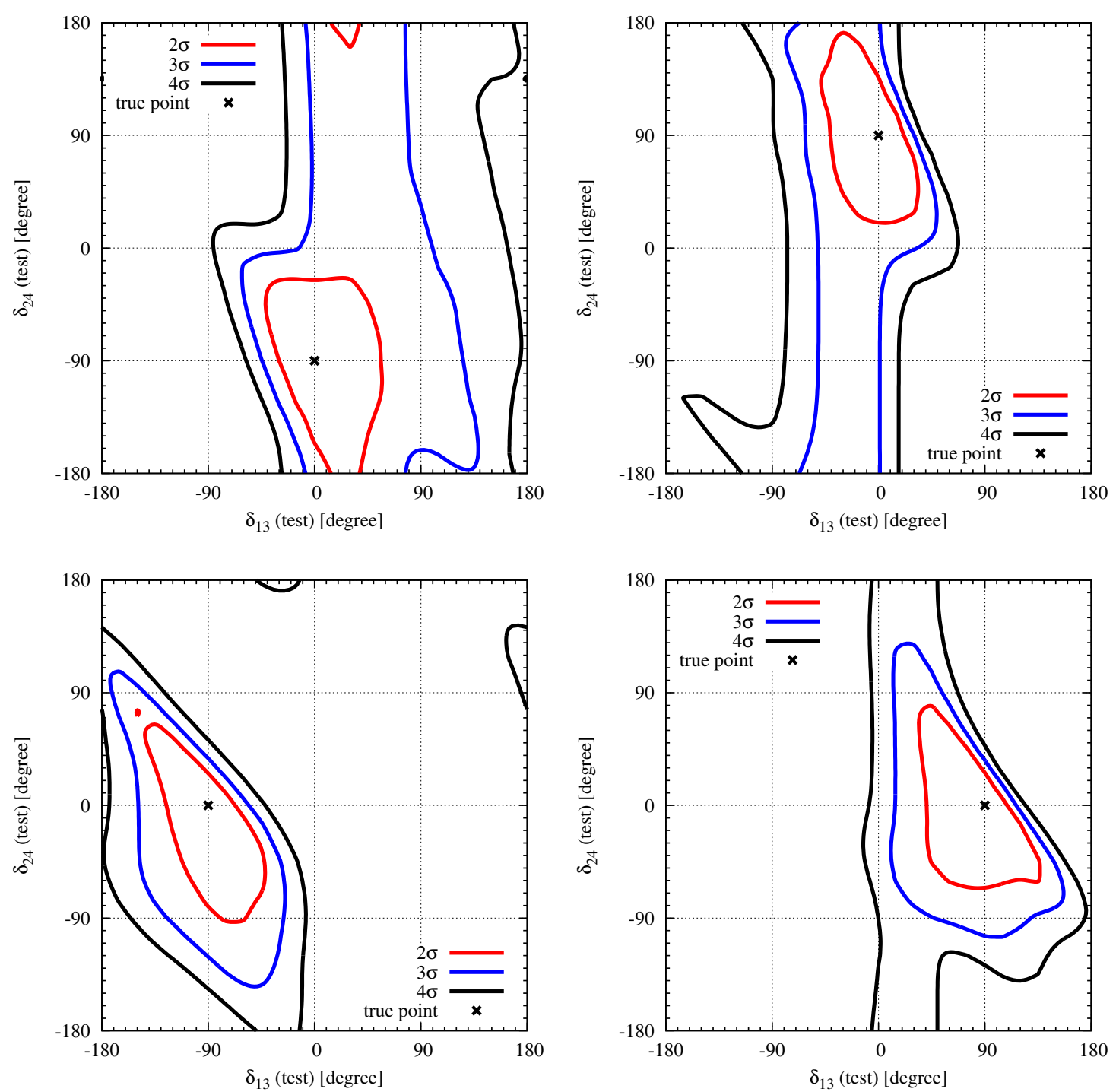

Figure 11. The allowed-region contours in the test $\delta_{13}$ - test $\delta_{24}$ plane for DUNE. True $\left(\theta_{14}, \theta_{24}, \theta_{34}\right)$ were taken to be $\left(12^{\circ}, 7^{\circ}, 25^{\circ}\right)$ and true $\delta_{34}=0$. Results have been shown for true $\left(\delta_{13}, \delta_{24}\right)=$ $\left(0, \pm 90^{\circ}\right)$ and $\left( \pm 90^{\circ}, 0\right) .2 \sigma, 3 \sigma$, and $4 \sigma$ results (corresponding to $\Delta \chi_{\min }^{2}=6.18,11.83$ and 19.33 respectively for two degrees of freedom) have been shown. The true point in each plot has been shown with a cross.

The top panels of figure 11 correspond to the choice of true $\left(\delta_{13}, \delta_{24}\right)$ being $\left(0,-90^{\circ}\right)$ (left) and $\left(0,90^{\circ}\right)$ (right). In the left panel, we see that test values of $\left(\delta_{13}, \delta_{24}\right)$ close to $\left(90^{\circ}, 0\right)$ and $\left(90^{\circ},-180^{\circ}\right)$ are allowed within $3 \sigma$ contours. Thus, it might be concluded that attributing $\mathrm{CP}$ violation unambiguously to $\delta_{24}$ may not be possible at $3 \sigma$. In the right panel, we see that test $\left(\delta_{13}, \delta_{24}\right)$ close to $\left(-90^{\circ}, 0\right)$ and $\left(-90^{\circ}, 180^{\circ}\right)$ are allowed, but at $4 \sigma$. Similar conclusions can also be drawn for the plots in the bottom panel where distinguishing maximally CP-violating $\delta_{13}$ and $\mathrm{CP}$ conserving $\delta_{24}$ from maximally $\mathrm{CP}$-violating $\delta_{24}$ and CP conserving $\delta_{13}$ may not be possible at $4 \sigma$. 


\subsection{How large do active-sterile mixings need to be before DUNE becomes sensitive to their presence?}

The Short Baseline Neutrino (SBN) program at Fermilab aims to conclusively establish the existence or else to place stringent constraints on the possible existence of the sterile neutrinos. At short baselines, the $P_{\mu e}$ oscillation probability is sensitive only to the masssquared difference $\delta m_{41}^{2}$ and an effective mixing angle given by $\sin ^{2} 2 \theta_{\mu e}=\sin ^{2} 2 \theta_{14} \sin ^{2} \theta_{24}$. For $\delta m_{41}^{2} \sim 1 \mathrm{eV}^{2}$ induced oscillations, the SBN program can exclude at $3 \sigma$ only $\sin ^{2} 2 \theta_{\mu e} \geq$ 0.001 [35]. It is natural to ask how tightly active-sterile mixings need to be excluded to ensure that DUNE measurements can be safely interpreted without taking the possible existence of sterile neutrinos into account. Phrasing this question another way, we ask whether active-sterile mixings corresponding to $\sin ^{2} 2 \theta_{\mu e}<0.001$ can be detected by the DUNE far detector. Figure 12 (left panel) throws some light on this question. Here, we have compared the $\mathrm{CP}$ bands in event rates assuming $3+1$ and $3+0$ for very small mixing angles $-\theta_{14}, \theta_{24}, \theta_{34}=3^{\circ}, 2^{\circ}, 10^{\circ}\left(\sin ^{2} 2 \theta_{\mu e} \approx 0.00001\right)$. We see that the $3+1$ band is completely degenerate with the $3+0$ band (incorporating the errorbars ${ }^{9}$ ). Thus, for such small mixing angles, it seems that neither the Short Baseline experiments nor the DUNE experiment may see evidence of new physics attributable to sterile neutrinos. In the right panel of the same figure, however, we have chosen $\theta_{14}, \theta_{24}, \theta_{34}=10^{\circ}, 5^{\circ}, 20^{\circ}\left(\sin ^{2} 2 \theta_{\mu e} \approx 0.0009\right)$. These larger values, which correspond to an effective mixing angle at the $3 \sigma$ sensitivity of the short-baseline experiments, lead to enhanced effects. The grey band now extends significantly beyond the expected event rates for $3+0$, even after accounting for errors. This provides a suggestive estimate of how large the mixing angles need to be before sterile neutrino effects at DUNE start to be discernable.

We stress that short-baseline experiments are significantly more sensitive, by design, to $\mathrm{CP}$ conserving oscillatory effects in $3+1$ compared to long-baseline experiments, and hence remain the definitive test by which presence or absence of sterile neutrinos can be established. Long-baseline experiments, however, can become sensitive to the presence of this sector if CPV is present. Moreover, matter enhances the effects of CP, further enabling these experiments to become, in a sense, complementary detectors of sterile neutrinos [27]. Signals that could possibly be interpreted as those for sterile neutrinos at long-baseline experiments like DUNE would, however, remain supportive rather than definitive evidence, because they could concievably be mimicked by other physics beyond the SM (see, for instance, $[67,68,70-80]$ for the effect of propagation NSI on long baselines, especially at DUNE in the context of CP Violation and Mass hierarchy).

\section{Conclusions and summary}

This work examines how sensitivities of long-baseline experiments to the $\mathrm{MH}$ and $\mathrm{CP}$ violation are affected and altered in the presence of a sterile neutrino. It attempts to quantitatively examine questions raised in [27]. While we use DUNE as our benchmark

\footnotetext{
${ }^{9}$ The error for each energy bin is the quadrature sum of the statistical ( $\sqrt{\text { Event no. }}$ ) and the systematic error $(2 \%)$ [69]. The value is an estimated expected value assuming the presence of a highly capable near-detector [69].
} 

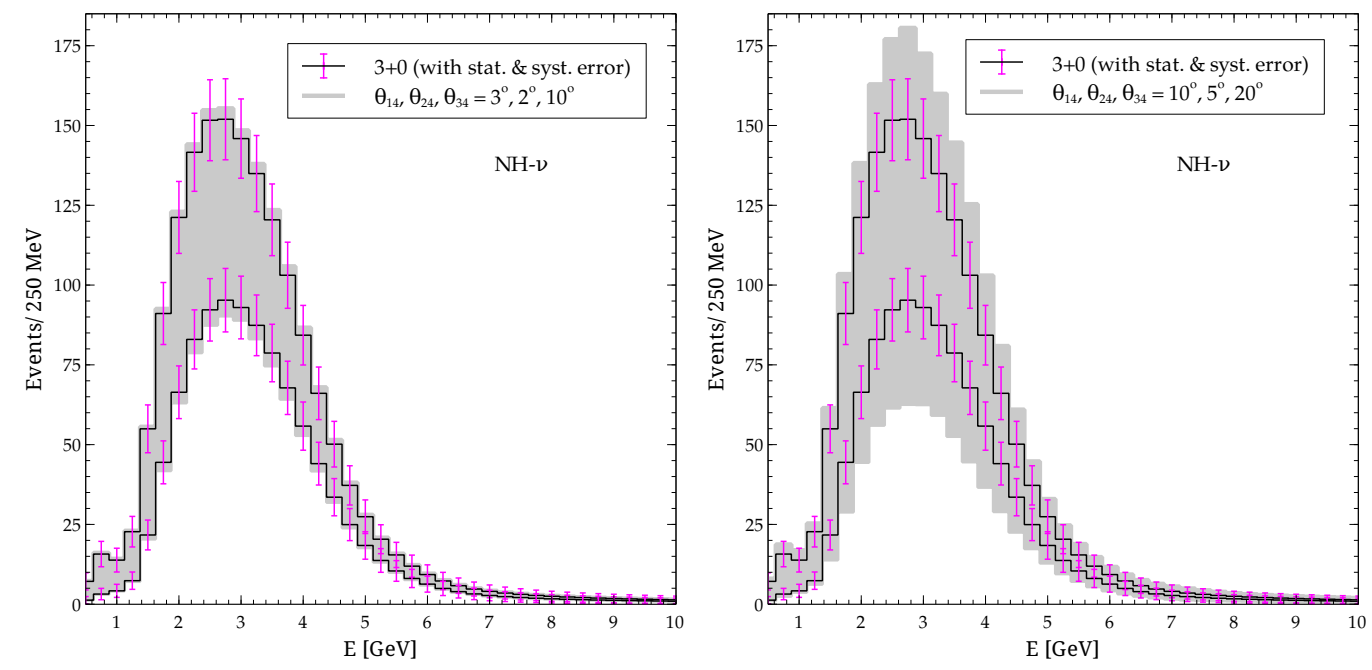

Figure 12. Neutrino event rates for the DUNE experiment as a function of the reconstructed neutrino energy. The black lines show the maximum and the minimum event rates corresponding to a variation of $\delta_{\mathrm{CP}}$ in $3+0$. Also shown are the corresponding statistical $(\sqrt{\text { Event no. }})$ and systematic error (2\%) [69] added in quadrature for each energy bin. The grey band corresponds to the maximum and minimum event rates assuming $3+1$ with $\theta_{14}, \theta_{24}, \theta_{34}=3^{\circ}, 2^{\circ}, 10^{\circ}$ (left panel), $\theta_{14}, \theta_{24}, \theta_{34}=10^{\circ}, 5^{\circ}, 20^{\circ}$ (right panel) and $\delta_{13}, \delta_{24}, \delta_{34}$ varied in $\left[-180^{\circ}, 180^{\circ}\right]$. Only the channel $\nu_{\mu} \rightarrow \nu_{e}$ has been considered with 5 years of $\nu$-running assuming normal hierarchy.

example, we study these sensitivities for T2K, HK, and NOvA also. Depending on the values of sterile mixing angles and phases, the sensitivities can be both significantly enhanced or suppressed compared to the $3+0$ case. We examine and discuss the reasons for this behaviour using the total event rate as a tool.

We also examine the ability of DUNE to pinpoint the origin of CPV, if such violation is detected by it. We find that while the discovery potential for the violation is large, determining its origin (i.e. ascribing it unambiguously to either the $3+0$ phase $\delta_{\mathrm{CP}}$ or one of the other phases $\delta_{24}, \delta_{34}$, present in $3+1$ ) is much more challenging. Indeed, $3 \sigma$ determination of the phase (or phases) responsible for CPV could prove very elusive both if sterile neutrinos are shown to exist, or if their existence cannot be conclusively ruled out by the short-baseline experiments. If the latter is the case, we ask how tightly one must then bound the sterile-active mixing angles to ensure that DUNE data can be safely interpreted without taking the possible existence of sterile neutrinos into account. In the process, we find that DUNE may exhibit signals hinting at the presence of a sterile sector even if the relevant mixing angles lie below the sensitivity of the planned short-baseline experiments. However, the ability of long-baseline efforts like DUNE to signal the presence of this sector, while highly valuable, must remain complementary to an essential and primary short-baseline thrust aimed at discovering evidence of short-wavelength oscillations with convincing redundancy. As emphasized both in [27] and this work, the sensitivity of DUNE to sterile neutrinos has qualitatively different origins and depends on interference effects and the matter enhancement of the corresponding mixing angles and $\mathrm{CP}$ violating phases. These are certainly aspects which do not lie within the physics ambit of short- 
baseline experiments; however, the resulting signals could also be mimicked by other new physics, preventing their unambiguous interpretation if considered in isolation.

In summary, DUNE provides strong and valuable complementarity in the search for a sterile sector to be conducted by the short-baseline experiments. It would be useful, in our view, to incorporate this capability into the thinking and efforts currently underway towards optimizing its design.

\section{Acknowledgments}

We gratefully acknowledge many helpful discussions on DUNE fluxes and errors with Mary Bishai and Elizabeth Worcester. We are also thankful to Dan Cherdack, Georgia Karagiorgi, Bryce Littlejohn, Poonam Mehta and Lisa Whitehead for very useful discussions. We also acknowledge the use of the HPC cluster facility at HRI for carrying out the numerical computations used in this work. RG acknowledges support and hospitality from the Neutrino and Theory Divisions at Fermilab while this work was in progress. DD, RG and MM acknowledge support from the DAE Neutrino project at HRI. Fermilab is operated by Fermi Research Alliance, LLC under contract no. DE-AC02-07CH11359 with the US Department of Energy.

Open Access. This article is distributed under the terms of the Creative Commons Attribution License (CC-BY 4.0), which permits any use, distribution and reproduction in any medium, provided the original author(s) and source are credited.

\section{References}

[1] Z. Maki, M. Nakagawa and S. Sakata, Remarks on the unified model of elementary particles, Prog. Theor. Phys. 28 (1962) 870 [inSPIRE].

[2] B. Pontecorvo, Neutrino Experiments and the Problem of Conservation of Leptonic Charge, Sov. Phys. JETP 26 (1968) 984 [Zh. Eksp. Teor. Fiz. 53 (1967) 1717] [InSPIRE].

[3] V.N. Gribov and B. Pontecorvo, Neutrino astronomy and lepton charge, Phys. Lett. B 28 (1969) 493 [INSPIRE].

[4] Super-Kamiokande collaboration, Y. Ashie et al., Evidence for an oscillatory signature in atmospheric neutrino oscillation, Phys. Rev. Lett. 93 (2004) 101801 [hep-ex/0404034] [INSPIRE].

[5] Super-Kamiokande collaboration, R. Wendell et al., Atmospheric neutrino oscillation analysis with sub-leading effects in Super-Kamiokande I, II and III, Phys. Rev. D 81 (2010) 092004 [arXiv: 1002 .3471] [INSPIRE].

[6] SNO collaboration, B. Aharmim et al., Electron energy spectra, fluxes and day-night asymmetries of ${ }^{8} \mathrm{~B}$ solar neutrinos from measurements with $\mathrm{NaCl}$ dissolved in the heavy-water detector at the Sudbury Neutrino Observatory, Phys. Rev. C 72 (2005) 055502 [nucl-ex/0502021] [INSPIRE].

[7] K2K collaboration, M.H. Ahn et al., Measurement of Neutrino Oscillation by the K2K Experiment, Phys. Rev. D 74 (2006) 072003 [hep-ex/0606032] [INSPIRE]. 
[8] MINOS collaboration, D.G. Michael et al., Observation of muon neutrino disappearance with the MINOS detectors and the NuMI neutrino beam, Phys. Rev. Lett. 97 (2006) 191801 [hep-ex/0607088] [INSPIRE].

[9] MINOS collaboration, P. Adamson et al., A Study of Muon Neutrino Disappearance Using the Fermilab Main Injector Neutrino Beam, Phys. Rev. D 77 (2008) 072002 [arXiv:0711.0769] [INSPIRE].

[10] T2K collaboration, K. Abe et al., Observation of Electron Neutrino Appearance in a Muon Neutrino Beam, Phys. Rev. Lett. 112 (2014) 061802 [arXiv:1311.4750] [INSPIRE].

[11] T2K collaboration, K. Abe et al., Precise Measurement of the Neutrino Mixing Parameter $\theta_{23}$ from Muon Neutrino Disappearance in an Off-Axis Beam, Phys. Rev. Lett. 112 (2014) 181801 [arXiv: 1403.1532] [INSPIRE].

[12] KamLAND collaboration, T. Araki et al., Measurement of neutrino oscillation with KamLAND: Evidence of spectral distortion, Phys. Rev. Lett. 94 (2005) 081801 [hep-ex/0406035] [INSPIRE].

[13] Borexino collaboration, C. Arpesella et al., Direct Measurement of the ${ }^{7}$ Be Solar Neutrino Flux with 192 Days of Borexino Data, Phys. Rev. Lett. 101 (2008) 091302 [arXiv: 0805.3843] [INSPIRE].

[14] DAYA BAY collaboration, F.P. An et al., Observation of electron-antineutrino disappearance at Daya Bay, Phys. Rev. Lett. 108 (2012) 171803 [arXiv:1203.1669] [InSPIRE].

[15] RENO collaboration, J.K. Ahn et al., Observation of Reactor Electron Antineutrino Disappearance in the RENO Experiment, Phys. Rev. Lett. 108 (2012) 191802 [arXiv: 1204.0626] [INSPIRE].

[16] Double CHOOZ collaboration, Y. Abe et al., Reactor electron antineutrino disappearance in the Double CHOOZ experiment, Phys. Rev. D 86 (2012) 052008 [arXiv:1207.6632] [INSPIRE].

[17] G.L. Fogli, E. Lisi, A. Marrone, D. Montanino, A. Palazzo and A.M. Rotunno, Global analysis of neutrino masses, mixings and phases: entering the era of leptonic CP-violation searches, Phys. Rev. D 86 (2012) 013012 [arXiv:1205.5254] [InSPIRE].

[18] M.C. Gonzalez-Garcia, M. Maltoni and T. Schwetz, Global Analyses of Neutrino Oscillation Experiments, Nucl. Phys. B 908 (2016) 199 [arXiv:1512.06856] [InSPIRE].

[19] D.V. Forero, M. Tortola and J.W.F. Valle, Neutrino oscillations refitted, Phys. Rev. D 90 (2014) 093006 [arXiv: 1405.7540] [INSPIRE].

[20] G. Altarelli, Status of Neutrino Mass and Mixing, Int. J. Mod. Phys. A 29 (2014) 1444002 [arXiv: 1404.3859] [INSPIRE].

[21] LSND collaboration, A.A. Aguilar-Arevalo et al., Evidence for neutrino oscillations from the observation of $\bar{\nu}_{e}$ appearance in a $\bar{\nu}_{\mu}$ beam, Phys. Rev. D 64 (2001) 112007 [hep-ex/0104049] [INSPIRE].

[22] MiniBoonE collaboration, A.A. Aguilar-Arevalo et al., Unexplained Excess of Electron-Like Events From a 1-GeV Neutrino Beam, Phys. Rev. Lett. 102 (2009) 101802 [arXiv: 0812.2243] [INSPIRE].

[23] G. Mention et al., The Reactor Antineutrino Anomaly, Phys. Rev. D 83 (2011) 073006 [arXiv: 1101.2755] [INSPIRE]. 
[24] T.A. Mueller et al., Improved Predictions of Reactor Antineutrino Spectra, Phys. Rev. C 83 (2011) 054615 [arXiv: 1101.2663] [INSPIRE].

[25] MiniBoonE collaboration, A.A. Aguilar-Arevalo et al., Improved Search for $\bar{\nu}_{\mu} \rightarrow \bar{\nu}_{e}$ Oscillations in the MiniBooNE Experiment, Phys. Rev. Lett. 110 (2013) 161801 [arXiv: 1207.4809] [INSPIRE].

[26] N. Klop and A. Palazzo, Imprints of CP-violation induced by sterile neutrinos in T2K data, Phys. Rev. D 91 (2015) 073017 [arXiv:1412.7524] [INSPIRE].

[27] R. Gandhi, B. Kayser, M. Masud and S. Prakash, The impact of sterile neutrinos on CP measurements at long baselines, JHEP 11 (2015) 039 [arXiv:1508.06275] [INSPIRE].

[28] NOvA collaboration, D.S. Ayres et al., The NOvA Technical Design Report, FERMILAB-DESIGN-2007-01 [INSPIRE].

[29] J.L. Hewett et al., Fundamental Physics at the Intensity Frontier, arXiv:1205.2671 [doi:10.2172/1042577] [INSPIRE].

[30] LBNE collaboration, C. Adams et al., The Long-Baseline Neutrino Experiment: Exploring Fundamental Symmetries of the Universe, arXiv:1307.7335 [INSPIRE] and online pdf version at http://www.osti.gov/scitech/biblio/1128102.

[31] Hyper-Kamiokande Proto-Collaboration collaboration, K. Abe et al., Physics potential of a long-baseline neutrino oscillation experiment using a J-PARC neutrino beam and Hyper-Kamiokande, Prog. Theor. Exp. Phys. 2015 (2015) 053C02 [arXiv:1502.05199] [INSPIRE].

[32] LAR1-ND, ICARUS-WA104 and MicroBooNE collaborations, M. Antonello et al., A Proposal for a Three Detector Short-Baseline Neutrino Oscillation Program in the Fermilab Booster Neutrino Beam, arXiv:1503.01520 [INSPIRE].

[33] MicroBoone, ICARUS and SBND collaborations, J. Zennamo, The Short-Baseline Neutrino Program, in proceedings of the 48th Annual Fermilab User's Meeting, Fermilab, Batavia, Illinois, U.S.A., June 9-11 2015 and online pdf version at https://indico.fnal.gov/ getFile.py $/$ access? contribId $=54 \&$ sessionId $=23 \&$ resId $=0 \&$ materialId $=$ slides \&confId $=8982$.

[34] D. Garcia-Gamez, Short-Baseline Neutrino Oscillation Program at Fermilab, The University of Manchester (2016) and online pdf version at http://indico.hep.manchester.ac.uk/getFile. py $/$ access?contribId $=156 \&$ sessionId $=6 \&$ resId $=0 \&$ materialId $=$ slides\&confId $=4534$.

[35] L. Camilleri, The Fermilab short-baseline neutrino program, AIP Conf. Proc. 1680 (2015) 020004 [INSPIRE].

[36] A. Donini, M. Lusignoli and D. Meloni, Telling three neutrinos from four neutrinos at the neutrino factory, Nucl. Phys. B 624 (2002) 405 [hep-ph/0107231] [INSPIRE].

[37] A. Dighe and S. Ray, Signatures of heavy sterile neutrinos at long baseline experiments, Phys. Rev. D 76 (2007) 113001 [arXiv:0709.0383] [INSPIRE].

[38] A. Donini, K.-i. Fuki, J. Lopez-Pavon, D. Meloni and O. Yasuda, The Discovery channel at the Neutrino Factory: $\nu_{\mu} \rightarrow \nu_{\tau}$ pointing to sterile neutrinos, JHEP 08 (2009) 041 [arXiv: 0812.3703] [INSPIRE].

[39] O. Yasuda, Sensitivity to sterile neutrino mixings and the discovery channel at a neutrino factory, arXiv: 1004.2388 [INSPIRE]. 
[40] D. Meloni, J. Tang and W. Winter, Sterile neutrinos beyond LSND at the Neutrino Factory, Phys. Rev. D 82 (2010) 093008 [arXiv: 1007. 2419] [InSPIRE].

[41] B. Bhattacharya, A.M. Thalapillil and C.E.M. Wagner, Implications of sterile neutrinos for medium/long-baseline neutrino experiments and the determination of $\theta_{13}$, Phys. Rev. D 85 (2012) 073004 [arXiv:1111.4225] [INSPIRE].

[42] MINOS collaboration, P. Adamson et al., Combined analysis of $\nu_{\mu}$ disappearance and $\nu_{\mu} \rightarrow \nu_{e}$ appearance in MINOS using accelerator and atmospheric neutrinos, Phys. Rev. Lett. 112 (2014) 191801 [arXiv:1403.0867] [INSPIRE].

[43] D. Hollander and I. Mocioiu, Minimal $3+2$ sterile neutrino model at LBNE, Phys. Rev. D 91 (2015) 013002 [arXiv: 1408.1749] [INSPIRE].

[44] J.M. Berryman, A. de Gouvêa, K.J. Kelly and A. Kobach, Sterile neutrino at the Deep Underground Neutrino Experiment, Phys. Rev. D 92 (2015) 073012 [arXiv:1507.03986] [INSPIRE].

[45] S.K. Agarwalla, S.S. Chatterjee, A. Dasgupta and A. Palazzo, Discovery Potential of T2K and NOvA in the Presence of a Light Sterile Neutrino, JHEP 02 (2016) 111 [arXiv: 1601.05995] [INSPIRE].

[46] S.K. Agarwalla, S.S. Chatterjee and A. Palazzo, Physics Reach of DUNE with a Light Sterile Neutrino, JHEP 09 (2016) 016 [arXiv: 1603.03759] [INSPIRE].

[47] M.A. Acero, A.A. Aguilar-Arevalo and J.C. D'Olivo, Earth matter effect on active-sterile neutrino oscillations, J. Phys. Conf. Ser. 315 (2011) 012015 [INSPIRE].

[48] P. Huber, M. Lindner and W. Winter, Simulation of long-baseline neutrino oscillation experiments with GLoBES (General Long Baseline Experiment Simulator), Comput. Phys. Commun. 167 (2005) 195 [hep-ph/0407333] [INSPIRE].

[49] P. Huber, J. Kopp, M. Lindner, M. Rolinec and W. Winter, New features in the simulation of neutrino oscillation experiments with GLoBES 3.0: General Long Baseline Experiment Simulator, Comput. Phys. Commun. 177 (2007) 432 [hep-ph/0701187] [INSPIRE].

[50] J. Kopp, Sterile neutrinos and non-standard neutrino interactions in GLoBES, (2010) https://www.mpi-hd.mpg.de/personalhomes/globes/tools/snu-1.0.pdf.

[51] J. Kopp, M. Lindner, T. Ota and J. Sato, Non-standard neutrino interactions in reactor and superbeam experiments, Phys. Rev. D 77 (2008) 013007 [arXiv: 0708.0152] [InSPIRE].

[52] M.C. Gonzalez-Garcia, M. Maltoni and T. Schwetz, Updated fit to three neutrino mixing: status of leptonic CP-violation, JHEP 11 (2014) 052 [arXiv:1409.5439] [INSPIRE].

[53] F. Capozzi, G.L. Fogli, E. Lisi, A. Marrone, D. Montanino and A. Palazzo, Status of three-neutrino oscillation parameters, circa 2013, Phys. Rev. D 89 (2014) 093018 [arXiv: 1312.2878] [INSPIRE].

[54] A. Esmaili, E. Kemp, O.L.G. Peres and Z. Tabrizi, Probing light sterile neutrinos in medium baseline reactor experiments, Phys. Rev. D 88 (2013) 073012 [arXiv:1308.6218] [INSPIRE].

[55] J. Kopp, P.A.N. Machado, M. Maltoni and T. Schwetz, Sterile Neutrino Oscillations: The Global Picture, JHEP 05 (2013) 050 [arXiv:1303.3011] [INSPIRE].

[56] Daya Bay collaboration, F.P. An et al., Search for a Light Sterile Neutrino at Daya Bay, Phys. Rev. Lett. 113 (2014) 141802 [arXiv: 1407.7259] [INSPIRE]. 
[57] Y. Declais et al., Search for neutrino oscillations at 15-meters, 40-meters and 95-meters from a nuclear power reactor at Bugey, Nucl. Phys. B 434 (1995) 503 [INSPIRE].

[58] B. Jones, Results of the Search for Sterile Neutrinos with IceCube, talk given at FermiLab, February 122016 and online pdf version at https://hep.uchicago.edu/seminars/semwin2016/BenJones1.pdf.

[59] MINOS collaboration, P. Adamson et al., Active to sterile neutrino mixing limits from neutral-current interactions in MINOS, Phys. Rev. Lett. 107 (2011) 011802 [arXiv:1104.3922] [INSPIRE].

[60] M. Bass et al., Baseline Optimization for the Measurement of CP-violation, Mass Hierarchy and $\theta_{23}$ Octant in a Long-Baseline Neutrino Oscillation Experiment, Phys. Rev. D 91 (2015) 052015 [arXiv: 1311.0212] [INSPIRE].

[61] T2K collaboration, Y. Itow et al., The JHF-Kamioka neutrino project, in proceedings of the $3 r d$ Workshop on Neutrino Oscillations and Their Origin (NOON 2001), Kashiwa, Japan, December 5-8 2001, pp. 239-248 [hep-ex/0106019] [INSPIRE].

[62] M. Ishitsuka, T. Kajita, H. Minakata and H. Nunokawa, Resolving neutrino mass hierarchy and CP degeneracy by two identical detectors with different baselines, Phys. Rev. D 72 (2005) 033003 [hep-ph/0504026] [INSPIRE].

[63] S.K. Agarwalla, S. Prakash, S.K. Raut and S.U. Sankar, Potential of optimized NOvA for large $\theta_{13} \& 3$ combined performance with a LArTPC \& T2K, JHEP 12 (2012) 075 [arXiv: 1208.3644] [INSPIRE].

[64] DUNE collaboration, R. Acciarri et al., Long-Baseline Neutrino Facility (LBNF) and Deep Underground Neutrino Experiment (DUNE), arXiv:1601.02984 [INSPIRE].

[65] B. Choudhary, R. Gandhi, S.R. Mishra, S. Mishra and J. Strait, LBNE-INDIA. Proposal of Indian Institutions and Fermilab Collaboration for Participation in the Long-Baseline Neutrino Experiment at Fermilab (A Proposal to Design and Build a High-Resolution Near Detector and to Contribute to the Liquid Argon Far Detector) to Department of Atomic Energy and Department of Science and Technology Government of India, (2012) http://lbne2-docdb.fnal.gov/cgi-bin/RetrieveFile?docid=6704\&filename= LBNE-India-DPR-V12-Science.pdf\&version=1.

[66] S. Choubey and D. Pramanik, Constraints on Sterile Neutrino Oscillations using DUNE Near Detector, arXiv:1604.04731 [INSPIRE].

[67] M. Masud and P. Mehta, Nonstandard interactions spoiling the CP-violation sensitivity at DUNE and other long baseline experiments, Phys. Rev. D 94 (2016) 013014 [arXiv: 1603.01380] [INSPIRE].

[68] M. Masud and P. Mehta, Nonstandard interactions and resolving the ordering of neutrino masses at DUNE and other long baseline experiments, Phys. Rev. D 94 (2016) 053007 [arXiv: 1606.05662] [INSPIRE].

[69] E. Worcester, private communication (2016).

[70] M. Masud, A. Chatterjee and P. Mehta, Probing CP-violation signal at DUNE in presence of non-standard neutrino interactions, J. Phys. G 43 (2016) 095005 [arXiv:1510.08261] [INSPIRE].

[71] P. Coloma, Non-Standard Interactions in propagation at the Deep Underground Neutrino Experiment, JHEP 03 (2016) 016 [arXiv:1511.06357] [INSPIRE]. 
[72] A. de Gouvêa and K.J. Kelly, Non-standard Neutrino Interactions at DUNE, Nucl. Phys. B 908 (2016) 318 [arXiv:1511.05562] [INSPIRE].

[73] D.V. Forero and P. Huber, Hints for leptonic CP-violation or New Physics?, Phys. Rev. Lett. 117 (2016) 031801 [arXiv:1601.03736] [INSPIRE].

[74] J. Liao, D. Marfatia and K. Whisnant, Degeneracies in long-baseline neutrino experiments from nonstandard interactions, Phys. Rev. D 93 (2016) 093016 [arXiv:1601.00927] [INSPIRE].

[75] K. Huitu, T.J. Kärkkäinen, J. Maalampi and S. Vihonen, Constraining the nonstandard interaction parameters in long baseline neutrino experiments, Phys. Rev. D 93 (2016) 053016 [arXiv: 1601.07730] [INSPIRE].

[76] P. Bakhti and Y. Farzan, CP-Violation and Non-Standard Interactions at the MOMENT, JHEP 07 (2016) 109 [arXiv: 1602.07099] [INSPIRE].

[77] A. Rashed and A. Datta, Determination of mass hierarchy with $\nu_{\mu} \rightarrow \nu_{\tau}$ appearance and the effect of nonstandard interactions, arXiv:1603.09031 [INSPIRE].

[78] P. Coloma and T. Schwetz, Generalized mass ordering degeneracy in neutrino oscillation experiments, Phys. Rev. D 94 (2016) 055005 [arXiv: 1604.05772] [INSPIRE].

[79] A. de Gouvêa and K.J. Kelly, False Signals of CP-Invariance Violation at DUNE, arXiv: 1605.09376 [INSPIRE].

[80] M. Blennow, S. Choubey, T. Ohlsson, D. Pramanik and S.K. Raut, A combined study of source, detector and matter non-standard neutrino interactions at DUNE, JHEP 08 (2016) 090 [arXiv: 1606. 08851] [INSPIRE]. 